\title{
NEW FRACTIONAL PSEUDOSPECTRAL METHODS WITH ACCURATE CONVERGENCE RATES FOR FRACTIONAL DIFFERENTIAL EQUATIONS*
}

\author{
SHERVAN ERFANI $^{\dagger}$, ESMAIL BABOLIAN $^{\dagger}$, AND SHAHNAM JAVADI ${ }^{\dagger}$
}

\begin{abstract}
The main purpose of this paper is to introduce generalized fractional pseudospectral integration and differentiation matrices using a family of fractional interpolants, called fractional Lagrange interpolants. We develop novel approaches to the numerical solution of fractional differential equations with a singular behavior at an end-point. To achieve this goal, we present efficient and stable methods based on three-term recurrence relations, generalized barycentric representations, and Jacobi-Gauss quadrature rules to evaluate the corresponding matrices. In a special case, we prove the equivalence of the proposed fractional pseudospectral methods using a suitable fractional Birkhoff interpolation problem. In fact, the fractional integration matrix yields the stable inverse of the fractional differentiation matrix, and the resulting system is well-conditioned. We develop efficient implementation procedures for providing optimal error estimates with accurate convergence rates for the interpolation operators and the proposed schemes in the $L^{2}$-norm. Some numerical results are given to illustrate the accuracy and performance of the algorithms and the convergence rates.
\end{abstract}

Key words. convergence rate, fractional differential equations, fractional Birkhoff interpolation, fractional pseudospectral methods, fractional Lagrange interpolants, singularity

AMS subject classifications. 26A33, 41A05, 65M06, 65M12, 65L60

1. Introduction. Recently, fractional integral and differential models have attracted considerable interest due to their applications in the modeling of a variety of physical phenomena such as fluid-dynamics, viscoelastic materials, astronautics, signal processing, robotics, and economics, among others; see, e.g., [3, 5, 9, 19, 23, 37]. There are many different definitions of fractional operators, such as the Riemann-Liouville integral/derivative and the Caputo derivative. In the fractional models, the differential and integral operators of fractional order are nonlocal with weakly singular kernels. Hence, the solutions of fractional differential equations (FDEs) are generally nonsmooth near the boundaries of the domain. However, in the last few years, some numerical approaches have been proposed for solving FDEs, but the error analysis for the proposed methods has mainly focused on smooth solutions (see, e.g., $[18,22,24,30,34,39,40])$.

Inspired by the nonlocal property of the fractional operators, global methods such as pseudospectral methods have become increasingly popular for the numerical solution of fractional problems. The basis functions are typically Lagrange interpolation polynomials, and the collocation points are chosen based on Jacobi-Gauss-type quadrature rules. In pseudospectral methods, the problem is discretized into a finite-dimensional linear/nonlinear system using pseudospectral integration matrices (PIMs) and pseudospectral differentiation matrices (PDMs). Since the solutions of FDEs-even for well-behaved inputs-might contain some singular terms, spectral collocation methods with classical Lagrange interpolants cannot lead to exponential convergence [41, 42].

More recently, several numerical approaches have been presented to resolve the singularities of nonsmooth solutions of FDEs. Zayernouri and Karniadakis [39] introduced as fractional basis functions the so-called Jacobi poly-fractonomials (JPFs) to approximate such singular solutions for some simple model FDEs. Also, a new family of nodal basis functions called fractional Lagrange interpolants was used in [40] to define the corresponding fractional

*Received October 24, 2019. Accepted September 22, 2020. Published online on January 22, 2021. Recommended by JaEun Ku.

${ }^{\dagger}$ Faculty of Mathematical Sciences and Computer, Kharazmi University, 50 Taleghani Avenue, Tehran 1561836314, Iran (shahnam. javadi@gmail.com). 


\section{ETNA}

Kent State University and

Johann Radon Institute (RICAM)

spectral collocation method. Chen, Shen, and Wang [6] developed generalized Jacobi functions (GJFs) as basis functions, and they proposed error estimates with convergence rates in weighted Sobolev spaces for the truncated approximation. In [43], a unified numerical framework with optimal error estimates for spectral Petrov-Galerkin methods and spectral collocation methods was developed to solve linear FDEs with singular solutions. Also, the interpolation errors in nonuniform Jacobi-weighted Sobolev spaces for FDEs with the Caputo fractional derivative were presented in [12]. In [35], fractional pseudospectral integration matrices (FPIMs) were introduced using classical Lagrange interpolation polynomials to solve FDEs and fractional integro-differential equations. In [17] fractional differentiation matrices were proposed based on JPFs and Jacobi-Gauss-Lobatto (JGL) points to construct spectral collocation methods. Furthermore, the authors introduced optimal integration preconditioners for fractional collocation methods, which lead to well-conditioned systems. In [34], the integral and differential fractional pseudospectral schemes were developed using weighted Lagrange interpolation. Also, the authors provided the equivalence between the fractional pseudospectral methods from the perspective of fractional Birkhoff interpolation. In [44], a multi-domain spectral collocation method was developed for variable-order nonlinear fractional differential equations. Finally, it is also noteworthy to point out a recent interest in developing numerical methods for solving FDEs; see, e.g., $[13,21,38]$ and the references therein.

Most of the recent works on numerical methods for resolving the singularities of nonsmooth solutions of the considered FDEs are based on weighted basis spaces. It is worth noting that the introduced methods based on a weighted basis (for example JPFs and GJFs) can only separate the first singular factor. Moreover, numerical results show that the proposed convergence rates for existing methods are usually pessimistic (see [6, 17, 43]). Convergence rates presented in these papers are not well suited for problems with singular solutions and singular source terms. For example, the convergence rate in [6] is suitable only for a sufficiently smooth source term. In fact, the solution and source term may not be in the same type of Sobolev spaces; from this point of view, we propose that the spectral rate of convergence for the $L^{2}$-errors must be related to the singularities of the solution and the source term.

In this paper, we define a family of fractional interpolation functions that enable us to capture more singular factors of the true solution. The use of this kind of interpolant is motivated by the fact that the solutions or right-hand sides of the fractional problems may have two or more singular terms. Next, we briefly highlight the main contributions of our development:

(1) We introduce the generalized fractional pseudospectral integral and differentiation matrices (GFPIMs and GFPDMs) at a specified set of Jacobi-Gauss (JG) points and flipped Jacobi-Gauss-Radau (FJGR) points by employing fractional-power Lagrange interpolation functions. Then, we develop new integral/differential fractional pseudospectral schemes together with the implementation details for solving FDEs with singular solutions.

(2) We explore the relations between the mapped Jacobi polynomials and the fractionalpower Lagrange functions. Then we use JG-type quadrature and three-term formulas to present a special and smart way for computing the GFPIMs and GFPDMs efficiently and stably. Moreover, we present the generalized barycentric form to compute the proposed interpolants and the corresponding barycentric weights explicitly at the collocation points.

(3) We show that the submatrix of the GFPDM is invertible, and we provide a stable way to compute its inverse. In a special case, we also prove the equivalence between the proposed differential and the integral fractional pseudospectral schemes from the perspective of Caputo fractional Birkhoff interpolation. Therefore, we employ GFPIMs for preconditioning the resulting system of the generalized differential fractional pseudospectral method. 
(4) We define new generalized Lagrange interpolation operators in Müntz spaces and provide some inequalities to obtain optimal error estimates. A rigorous convergence analysis for the present fractional pseudospectral schemes in $L^{2}$-space is given. Moreover, for the first time, we present accurate convergence rates for the errors of the introduced interpolation operators and the numerical methods.

The rest of this paper is organized as follows. In the next section, a brief overview and some preliminaries are presented for the subsequent developments. In Section 3, we introduce fractional-power Lagrange interpolants and present the definitions and computation of GFPIMs and GFPDMs. In Section 4, using Caputo fractional Birkhoff interpolation, the equivalence mentioned above is proved. We focus on the implementation details of the new fractional pseudospectral methods to solve linear and nonlinear FDEs in Section 5. In Section 6, we apply the generalized Lagrange interpolants for fractional pseudospectral methods to derive error estimates and convergence rates. Numerical examples and applications are provided in Section 7 to illustrate the effectiveness and accuracy of the proposed methods and the convergence rates. We conclude and discuss our approaches in the final section.

2. Preliminaries. In this section, we state some necessary preparations and definitions for subsequent discussions. We present the definitions of fractional integrals and derivatives. We then collect some important properties and useful relations of the Müntz-Legendre polynomials.

2.1. Fractional integrals and derivatives. Let $A C[a, b]$ be the space of functions that are absolutely continuous on $[a, b]$. For $m \in \mathbb{N}:=\{1,2, \ldots\}$, the space $A C^{m}[a, b]$ is defined by

$$
A C^{m}[a, b]=\left\{u:[a, b] \longrightarrow \mathbb{R} \mid D^{m-1} f \in A C^{m}[a, b]\right\}
$$

DEFINITION 2.1. The left Riemann-Liouville fractional integral (RLFI) of real order $\alpha>0$ of a function $u$ is defined as

$$
{ }_{a} I_{t}^{\alpha} u(t)=\frac{1}{\Gamma(\alpha)} \int_{a}^{t}(t-s)^{\alpha-1} u(s) d s, \quad t \in[a, b],
$$

and the left Caputo fractional derivative (CFD) of real order $\alpha$ of a function $u \in A C^{m}[a, b]$ is defined as ${ }_{a}^{c} D_{t}^{\alpha} u(t)={ }_{a} I_{t}^{m-\alpha}\left(\frac{d^{m}}{d t^{m}} u(t)\right)$, where $m=\lceil\alpha\rceil \in \mathbb{N}$ is the smallest integer greater than or equal to $\alpha$ [19].

The left RLFI and left CFD operators applied to power functions return power functions of the same form. Let $\alpha>0$ and $\beta>-1$. Then

$$
{ }_{a} I_{t}^{\alpha}(t-a)^{\beta}=\frac{\Gamma(\beta+1)}{\Gamma(\beta+\alpha+1)}(t-a)^{\beta+\alpha},
$$

and for $\alpha \in(m-1, m)$ with $m \in \mathbb{N}$,

$$
{ }_{a}^{c} D_{t}^{\alpha}(t-a)^{\beta}= \begin{cases}0, & \beta \in\{0,1, \ldots, m-1\}, \\ \frac{\Gamma(\beta+1)}{\Gamma(\beta-\alpha+1)}(t-a)^{\beta-\alpha}, & \beta>m-1, \beta \in \mathbb{R} .\end{cases}
$$

Moreover, if $u \in \mathbb{C}^{m}[a, b]$ and $\alpha \geq \beta$, then it holds that

$$
{ }_{a} I_{t}^{\beta}\left({ }_{a}^{c} D_{t}^{\alpha} u(t)\right)={ }_{a}^{c} D_{t}^{\alpha-\beta} u(t)-\sum_{k=\lceil\alpha-\beta\rceil}^{m-1} \frac{u^{(k)}(a)}{\Gamma(k-(\alpha-\beta)+1)}(t-a)^{k-(\alpha-\beta)} .
$$


2.2. The Müntz-Legendre polynomials. The Müntz-Legendre (M-L) polynomials on the interval $\Omega=\left[0, t_{f}\right]$ are given by the formulas (see [29])

$$
L_{k}(t ; \mu):=J_{k}^{0, \frac{1}{\mu}-1}\left(2\left(\frac{t}{t_{f}}\right)^{\mu}-1\right)=\sum_{i=0}^{k} C_{k, i}\left(\frac{t}{t_{f}}\right)^{i \mu},
$$

where $J_{k}^{0, \frac{1}{\mu}-1}($.$) is the Jacobi polynomial with index \left(0, \frac{1}{\mu}-1\right)$ and

$$
C_{k, i}=\frac{(-1)^{k-i}}{\mu^{k} i !(k-i) !} \prod_{j=0}^{k-1}((i+j) \mu+1) .
$$

A direct consequence of [4, Corollary 3.6] and [28, Theorem 3.2] is the orthogonality of $\left\{L_{k}(t ; \mu)\right\}_{k=0}^{\infty}$ in $L^{2}(\Omega)$, i.e,

$$
\int_{0}^{t_{f}} L_{k}(t ; \mu) L_{j}(t ; \mu) d x=\frac{t_{f}}{2 k \mu+1} \delta_{j k}, \quad k, j=0,1, \ldots
$$

Since the coefficients $C_{k, i}$ grow fast with increasing $k$, a direct evaluation of M-L polynomials and their fractional integral/derivative by using the power form (2.2) can be problematic [15, 25, 28]. In order to compute M-L polynomials, we use a stable and efficient technique based on a three-term recurrence relation presented in [15]:

$$
\begin{aligned}
L_{0}(t ; \mu) & =1 \\
L_{1}(t ; \mu) & =\left(\frac{1}{\mu}+1\right)\left(\frac{t}{t_{f}}\right)^{\mu}-\frac{1}{\mu} \\
L_{k+1}(t ; \mu) & =\left(a_{k}\left(2\left(\frac{t}{t_{f}}\right)^{\mu}-1\right)-b_{k}\right) L_{k}(t ; \mu)-c_{k} L_{k-1}(t ; \mu), \quad k=1,2, \ldots,
\end{aligned}
$$

where

$$
\begin{array}{rlrl}
a_{k} & =\frac{\left(2 k+\frac{1}{\mu}\right)\left(2 k+\frac{1}{\mu}+1\right)}{2(k+1)\left(k+\frac{1}{\mu}\right)}, & b_{k}=\frac{\left(2 k+\frac{1}{\mu}\right)\left(\frac{1}{\mu}-1\right)^{2}}{2(k+1)\left(k+\frac{1}{\mu}\right)\left(2 k+\frac{1}{\mu}-1\right)}, \\
c_{k} & =\frac{k\left(2 k+\frac{1}{\mu}\right)\left(k+\frac{1}{\mu}-1\right)}{(k+1)\left(k+\frac{1}{\mu}\right)\left(2 k+\frac{1}{\mu}-1\right)} .
\end{array}
$$

The following theorem in [14] shows the relationship between the M-L polynomials and their ordinary derivatives.

THEOREM 2.2. The derivative of order $m=1,2, \ldots$ of $s^{\mu} \mathcal{L}(s ; \mu)$, for $\mu \in(m-1, m)$, can be expressed as

$$
\partial_{s}^{m}\left(s^{\mu} \mathcal{L}(s ; \mu)\right)=s^{\mu-m} \mathcal{A L}(s ; \mu),
$$

where $\mathcal{A}=\prod_{i=0}^{m-1}(\mathbf{A}+(\mu-i) \mathbf{I}), \mathcal{L}(t ; \mu)=\left(L_{0}(t ; \mu), L_{1}(t ; \mu), \ldots, L_{n-1}(t ; \mu)\right)^{T}, \mathbf{I}$ is the identity matrix of order $n$, and

$$
\mathbf{A}=\left[\begin{array}{cccccc}
0 & 0 & 0 & 0 & \ldots & 0 \\
1 & \mu & 0 & 0 & \ldots & 0 \\
1 & 2 \mu+1 & 2 \mu & 0 & \ldots & 0 \\
1 & 2 \mu+1 & 4 \mu+1 & 3 \mu & \ldots & 0 \\
\vdots & \vdots & \vdots & & \ddots & \vdots \\
1 & 2 \mu+1 & 4 \mu+1 & \ldots & & (n-1) \mu
\end{array}\right]_{n \times n}
$$


3. Computation of the generalized fractional pseudospectral matrices. The fractional pseudospectral integration and differentiation matrices are the essential building blocks of collocation methods for solving fractional problems. In this section, efficient and stable numerical methods to compute the GFPIMs and GFPDMs with JG and FJGR points are presented.

3.1. Computation of the GFPIMs. Let the JG points $\left\{\hat{t}_{i}\right\}_{i=1}^{N}$ be the zeros of $J_{N}^{0,-1+\frac{1}{\mu}}$, with $-1=\hat{t}_{0}<\hat{t}_{1}<\ldots<\hat{t}_{N+1}=1$, and $\left\{\hat{\omega}_{i}\right\}_{i=1}^{N}$ be the corresponding quadrature weights. Also, let the FJGR points $\left\{\check{t}_{i}\right\}_{i=1}^{N-1}$ be the zeros of $J_{N-1}^{1,-1+\frac{1}{\mu}}$, with $-1=\check{t}_{0}<\check{t}_{1}<\ldots<$ $\check{t}_{N}=1$, and with $\left\{\check{\omega}_{i}\right\}_{i=1}^{N}$ the corresponding quadrature weights. We define the GFPIMs of order $(\alpha, \mu)$ for the JG points and FJGR points, respectively, as

$$
\begin{aligned}
& \hat{\mathbf{I}}_{k i}^{\alpha, \mu}={ }_{0} I_{\tau_{k}}^{\alpha} \hat{h}_{i}^{\mu}(\tau), k=1, \ldots, N+1, \quad i=1, \ldots, N, \\
& \check{\mathbf{I}}_{k i}^{\alpha, \mu}={ }_{0} I_{\check{\tau}_{k}}^{\alpha} \check{h}_{i}^{\mu}(\tau), \quad k, i=1, \ldots, N,
\end{aligned}
$$

where $\hat{\tau}_{k}=t_{f}\left(\frac{\hat{t}_{k}+1}{2}\right)^{\frac{1}{\mu}} \in\left[0, t_{f}\right]$ and $\check{\tau}_{k}=t_{f}\left(\frac{\check{t}_{k}+1}{2}\right)^{\frac{1}{\mu}} \in\left[0, t_{f}\right]$ are the mapped JG and FJGR points. Here, $\hat{h}_{i}^{\mu}, \check{h}_{i}^{\mu}$ with $\mu>0$ are fractional Lagrange functions associated with the interpolation points $\left\{\hat{\tau}_{i}\right\}_{i=1}^{N}$ and $\left\{\check{\tau}_{i}\right\}_{i=1}^{N}$, respectively, defined as

$$
\hat{h}_{i}^{\mu}(\tau)=\prod_{j=1, j \neq i}^{N} \frac{\tau^{\mu}-\hat{\tau}_{j}^{\mu}}{\hat{\tau}_{i}^{\mu}-\hat{\tau}_{j}^{\mu}}, \quad \check{h}_{i}^{\mu}(\tau)=\prod_{j=1, j \neq i}^{N} \frac{\tau^{\mu}-\check{\tau}_{j}^{\mu}}{\check{\tau}_{i}^{\mu}-\check{\tau}_{j}^{\mu}}, \quad i=1, \ldots, N .
$$

It can be seen that the above interpolation functions can be adapted to more singular factors, but classical Lagrange and weighted Lagrange interpolation can only deal with at most one singular factor of the functions (see also Remark 3.3).

In [2] it is shown that the barycentric Lagrange interpolation polynomials avoid the problem of underflow and overflow. Here, to evaluate $\hat{h}_{i}^{\mu}$ at the collocation points, we use the following stable form of the generalized barycentric Lagrange interpolation functions given by

$$
\hat{h}_{i}^{\mu}(\tau)=\frac{\frac{\hat{\xi}_{i}^{\mu}}{\tau^{\mu}-\hat{\tau}_{i}^{\mu}}}{\sum_{j=1}^{N} \frac{\hat{\xi}_{j}^{\mu}}{\tau^{\mu}-\hat{\tau}_{j}^{\mu}}}, \quad i=1, \ldots, N
$$

where $\hat{\xi}_{i}^{\mu}$ are the barycentric weights defined as

$$
\hat{\xi}_{i}^{\mu}=\frac{1}{\prod_{k=1, k \neq i}^{N}\left(\hat{\tau}_{i}^{\mu}-\hat{\tau}_{k}^{\mu}\right)}, \quad i=1, \ldots, N
$$

Similar results also holds for $\check{h}_{i}^{\mu}(\tau)$ with the barycentric weights $\check{\xi}_{i}^{\mu}$. The barycentric weights can be computed efficiently and stably by (see $[2,32])$

$$
\begin{array}{rlrl}
\hat{\xi}_{i}^{\mu} & =(-1)^{i+1} \frac{2}{T^{\mu}} \sqrt{\left(t_{f}^{\mu}-\hat{\tau}_{i}^{\mu}\right) \hat{\tau}_{i}^{\mu} \hat{\omega}_{i}}, & i=1, \ldots N, \\
\check{\xi}_{i}^{\mu} & =(-1)^{i+1} \sqrt{2 \frac{\check{\tau}_{i}^{\mu}}{t_{f}^{\mu}} \check{\omega}_{i}}, & i=1, \ldots N-1, \quad \text { and } \\
\check{\xi}_{N}^{\mu}=(-1)^{N+1} \sqrt{2 \check{\omega}_{i}} . &
\end{array}
$$


To compute the GFPIMs of $\hat{h}_{i}^{\mu}$ of order $\alpha>0$, we express the interpolation functions $\hat{h}_{i}^{\mu} \in \mathbf{M}_{N-1, \mu}:=\operatorname{span}\left\{1, t^{\mu}, \ldots, t^{(N-1) \mu}\right\}$ in terms of the M-L polynomials as

$$
\hat{h}_{i}^{\mu}(\tau)=\sum_{j=0}^{N-1} \hat{\lambda}_{j i} L_{j}(\tau ; \mu)
$$

where

$$
\hat{\lambda}_{j, i}=\frac{2 j \mu+1}{\mu 2^{\frac{1}{\mu}}} \hat{\omega}_{i} L_{j}\left(\hat{\tau}_{i} ; \mu\right) .
$$

Let $l_{\mu}$ be a non-negative integer parameter. We split the M-L polynomial $L_{j}(\tau, \mu)$ into $L_{j}(\tau, \mu)=L_{j, 1}(\tau, \mu)+L_{j, 2}(\tau, \mu)$ such that $L_{j, 2}(\tau, \mu)=\sum_{r=0}^{l_{\mu}} C_{j r}\left(\frac{\tau}{t_{f}}\right)^{r \mu}$. From Definition 2.1 and using JG quadrature formulas with nodes and weights $\left\{\theta_{i}^{\alpha-1, \mu}, \omega_{i}^{\alpha-1, \mu}\right\}$ relative to the Jacobi weight function $w(t)=(1-t)^{\alpha-1}(1+t)^{\mu}$, we obtain

$$
\begin{aligned}
{ }_{0} I_{\hat{\tau}_{k}}^{\alpha}\left(\tau^{\mu} L_{j 1}(\tau ; \mu)\right) & =\frac{1}{\Gamma(\alpha)} \int_{0}^{\hat{\tau}_{k}}\left(\hat{\tau}_{k}-s\right)^{\alpha-1} s^{\mu} L_{j 1}(s ; \mu) d s \\
& \approx \frac{1}{\Gamma(\alpha)}\left(\frac{\hat{\tau}_{k}}{2}\right)^{\alpha+\mu} \sum_{i=1}^{M} \omega_{i}^{\alpha-1, \mu} L_{j 1}\left(\frac{\hat{\tau}_{k}}{2}\left(\theta_{i}^{\alpha-1, \mu}+1\right) ; \mu\right) .
\end{aligned}
$$

By direct calculations, we get

$$
{ }_{0} I_{\hat{\tau}_{k}}^{\alpha}\left(\tau^{\mu} L_{j 2}(\tau ; \mu)\right)=\sum_{r=0}^{l_{\mu}} C_{j r} \frac{\Gamma(1+(r+1) \mu)}{t_{f}^{r \mu} \Gamma(1+(r+1) \mu+\alpha)} \hat{\tau}_{k}^{(r+1) \mu+\alpha} .
$$

From (3.2)-(3.3) and the three-term formula in (2.3), for $j=1,2, \ldots$, we have

$$
\begin{aligned}
{ }_{0} I_{\hat{\tau}_{k}}^{\alpha} L_{0}(t ; \mu)= & \frac{1}{\Gamma(\alpha+1)} \tau^{\alpha}, \\
{ }_{0} I_{\hat{\tau}_{k}}^{\alpha} L_{1}(\tau ; \mu)= & \left(\frac{1}{\mu}+1\right) \frac{\Gamma(1+\mu)}{t_{f}^{\mu} \Gamma(1+\mu+\alpha)} \tau^{\alpha+\mu}-\frac{1}{\mu \Gamma(\alpha+1)} \tau^{\alpha}, \\
{ }_{0} I_{\hat{\tau}_{k}}^{\alpha} L_{j+1}(t ; \mu)= & \frac{2}{t_{f}^{\mu}} a_{k}\left({ }_{0} I_{\hat{\tau}_{k}}^{\alpha} \tau^{\mu}\left(L_{j 1}(\tau ; \mu)+L_{j 2}(\tau ; \mu)\right)\right) \\
& \quad-\left(a_{j}+b_{j}\right)_{0} I_{\hat{\tau}_{k}}^{\alpha} L_{j}(\tau ; \mu)-c_{j 0} I_{\hat{\tau}_{k}}^{\alpha} L_{j-1}(\tau ; \mu) .
\end{aligned}
$$

Thus, we get

$$
{ }_{0} I_{\hat{\tau}_{k}}^{\alpha} \hat{h}_{i}^{\mu}(\tau)=\sum_{j=0}^{N-1} \hat{\lambda}_{j i 0} I_{\hat{\tau}_{k}}^{\alpha} L_{j}(\tau ; \mu) .
$$

Although we have focused on the computation of the fractional integral of $\hat{h}_{i}^{\mu}(\tau)$ using JG points, the same results can be obtained for $\check{h}_{i}^{\mu}(\tau)$ with FJGR points.

REMARK 3.1. It is worth noting that we can choose the parameter $l_{\mu}$ such that $L_{j}^{1}(\tau ; \mu)$ is smooth enough (e.g., $\left.l_{\mu} \mu \geq 1\right)$ and also that the coefficients matrices $C_{j s}$ in $L_{j}^{2}(\tau ; \mu)$ are not too large. This parameter must be selected in order to balance smoothness and stability. We can observe that the singularity of the integrand function in ${ }_{0} I_{\tau}^{\alpha} \tau^{\mu} L_{j}^{2}(\tau ; \mu)$ is $\tau^{\left(1+l_{\mu}\right) \mu}$, thus using the discussion in [14] and the proper value of $l_{\mu}$, the integration error has a convergence rate of $O\left(M^{-2\left(1+\left(2+l_{\mu}\right) \mu\right.}\right)$. 
3.2. Computation of the GFPDMs. We define the GFPDMs of order $\alpha \in(m-1, m)$ with the JG points $\left\{\hat{\tau}_{i}\right\}_{i=1}^{N}$ and $\hat{\tau}_{0}=0, \hat{\tau}_{N+1}=1$ as

$$
\hat{\mathbf{D}}_{k i}^{\alpha}= \begin{cases}-\sum_{j=1}^{N} \hat{\mathbf{D}}_{k j}^{\alpha}, & i=0, \\ { }_{0}^{c} D_{\hat{\tau}_{k}}^{\alpha} \hat{l}_{i}^{\alpha}(\tau), & i=1,2, \ldots, N,\end{cases}
$$

and with the FJGR points $\left\{\check{\tau}_{i}\right\}_{i=1}^{N}$ and $\hat{\tau}_{0}=0$ as

$$
\check{\mathbf{D}}_{k i}^{\alpha}= \begin{cases}-\sum_{j=1}^{N} \check{\mathbf{D}}_{k j}^{\alpha}, & i=0, \\ { }_{0}^{c} D_{\check{\tau}_{k}}^{\alpha} \check{l}_{i}^{\alpha}(\tau), & i=1,2, \ldots, N,\end{cases}
$$

where $\left(\hat{l}_{i}^{\alpha}, \check{l}_{i}^{\alpha} \in \mathbf{M}_{N, \alpha}\right)_{i=1}^{N}$ are fractional Lagrange interpolation functions related to the JG and FJGR points by

$$
\hat{l}_{i}^{\alpha}(\tau)=\left(\frac{\tau}{\hat{\tau}_{i}}\right)^{\alpha} \hat{h}_{i}^{\alpha}(\tau), \quad \check{l}_{i}^{\alpha}(\tau)=\left(\frac{\tau}{\check{\tau}_{i}}\right)^{\alpha} \check{h}_{i}^{\alpha}(\tau), \quad i=1, \ldots, N .
$$

We observe that the GFPDMs are rectangular matrices of order $N \times(N+1)$. Here, we focus on computing the GFPDMs using JG points, but the same idea can be applied for evaluating them for the FJGR points. We expand $\hat{h}_{i}^{\alpha}(\tau)$ in terms of M-L polynomials (3.1), and, using Definition 2.1 and Theorem 2.2, we obtain

$$
\begin{aligned}
\hat{\mathbf{D}}_{k i}^{\alpha} & ={ }_{0}^{c} D_{\hat{\tau}_{k}}^{\alpha} \hat{l}_{i}^{\alpha}(\tau)=\frac{1}{\Gamma(m-\alpha)} \int_{0}^{\hat{\tau}_{k}}\left(\hat{\tau}_{k}-s\right)^{m-\alpha-1} \partial_{s}^{m}\left(\hat{l}_{i}^{\alpha}(s)\right) d s \\
& =\sum_{j=0}^{N-1} \frac{\hat{\lambda}_{j i}}{\Gamma(m-\alpha) \hat{\tau}_{i}^{\alpha}} \int_{0}^{\hat{\tau}_{k}}\left(\hat{\tau}_{k}-s\right)^{m-\alpha-1} \partial_{s}^{m}\left(s^{\alpha} L_{j}(s ; \alpha)\right) d s \\
& =\sum_{j=0}^{N-1} \frac{\hat{\lambda}_{j i} \mathcal{A}_{j,:}}{\Gamma(m-\alpha) \hat{\tau}_{i}^{\alpha}} \int_{0}^{\hat{\tau}_{k}}\left(\hat{\tau}_{k}-s\right)^{m-\alpha-1} s^{\alpha-m} \mathcal{L}(s ; \alpha) d s,
\end{aligned}
$$

where $\mathcal{A}_{j,:}$ is the $j$ th row of the matrix $\mathcal{A}$. For the next discussions, the notation $X_{i: j,:}$ will be used to denote the rows $i$ through $j$ of the matrix $X$, while the notation $X_{:, i: j}$ is used for the columns $i$ through $j$ of the matrix $X$. By splitting the M-L polynomial $\mathcal{L}(s ; \alpha)$ into $\mathcal{L}(s ; \alpha)=\mathcal{L}^{1}(s ; \alpha)+C_{:, 0: l_{\alpha}} \psi(s)$ with $\psi(s)=\left(1,\left(\frac{s}{t_{f}}\right)^{\alpha}, \ldots,\left(\frac{s}{t_{f}}\right)^{l_{\alpha} \alpha}\right)^{T}$, we have

$$
\begin{array}{r}
\hat{\mathbf{D}}_{k i}^{\alpha}=\sum_{j=0}^{N-1} \frac{\hat{\lambda}_{j i} \mathcal{A}_{j,:}}{\Gamma(m-\alpha) \hat{\tau}_{i}^{\alpha}}\left(\int_{0}^{\hat{\tau}_{k}}\left(\hat{\tau}_{k}-s\right)^{m-\alpha-1} s^{\alpha-m} \mathcal{L}^{1}(s ; \alpha) d s\right. \\
\left.+C_{:, 0: l_{\alpha}} \int_{0}^{\hat{\tau}_{k}}\left(\hat{\tau}_{k}-s\right)^{m-\alpha-1} s^{\alpha-m} \psi(s) d s\right) .
\end{array}
$$

Let $\left\{\theta_{r}^{\alpha, m}, \omega_{r}^{\alpha, m}\right\}_{r=1}^{M}$ be the set of JG-type points and quadrature weights with respect to the Jacobi weight function $\omega^{m-\alpha-1, \alpha-m}$. Using Beta functions (see [19]) and JG-type quadrature, we get

$$
\hat{\mathbf{D}}_{k i}^{\alpha} \approx \sum_{j=0}^{N-1} \frac{\hat{\lambda}_{j i} \mathcal{A}_{j,:}}{\Gamma(m-\alpha) \hat{\tau}_{i}^{\alpha}}\left(\sum_{r=1}^{M} \omega_{r}^{\alpha, m} \mathcal{L}^{1}\left(\hat{\tau}_{k} \frac{1+\theta_{r}^{\alpha, m}}{2} ; \nu\right)+C_{:, 0: l_{\alpha}} \Psi\right),
$$




\section{ETNA}

Kent State University and

Johann Radon Institute (RICAM)

where $\Psi$ is a column vector with entries $\Psi_{i}=\frac{\hat{\tau}_{k}^{(i-1) \alpha} \Gamma(i \alpha-m+1) \Gamma(m-\alpha)}{t_{f}^{(i-1) \alpha} \Gamma((i-1) \alpha+1)}$, for $i=1, \ldots, l_{\alpha}+1$. The integration error of the JG-type quadrature in (3.6) has the rate $O\left(M^{-2\left(1+\left(2+l_{\alpha}\right) \alpha-m\right.}\right)$ (see [14]).

THEOREM 3.2. The matrices $\hat{\mathbf{D}}_{:, 1: N}^{\alpha}$ and $\check{\mathbf{D}}_{:, 1: N}^{\alpha}$ obtained by deleting the first column of $\hat{\mathbf{D}}^{\alpha}$ and $\check{\mathbf{D}}^{\alpha}$, respectively, are invertible.

Proof. For brevity, we just show that $\hat{\mathbf{D}}_{:, 1: N}^{\alpha}$ is invertible. The nonsingularity of $\check{\mathbf{D}}_{:, 1: N}^{\alpha}$ is established in a similar way.

Suppose that for some vector $w=\left(w_{1}, w_{2}, \ldots, w_{N}\right) \in \mathbb{R}^{N}$, we have $\hat{\mathbf{D}}_{:, 1: N}^{\alpha} w=0$. Let $w \in \mathbf{M}_{N, \alpha}$ be the unique function that satisfies $w\left(\hat{\tau}_{i}\right)=w_{i}, i=1, \ldots, N$. We have $0=\left(\hat{\mathbf{D}}_{:, 1: N}^{\alpha} w\right)_{i}={ }_{0}^{c} D_{\hat{\tau}_{i}}^{\alpha} w(\tau)$. Let $u(\tau)={ }_{0}^{c} D_{\tau}^{\alpha} w(\tau)$. Then $u \in \mathbf{M}_{N-1, \alpha}$ and $u\left(\hat{\tau}_{i}\right)=0$. Let $t=\tau^{\alpha}$. Then $u\left(t^{\frac{1}{\alpha}}\right)$ is a polynomial of degree at most $N-1$. It must be identically zero since it vanishes at the $N$ points $t_{i}=\hat{\tau}_{i}^{\alpha}$. Hence, it follows that $u$ is identically 0 . This shows that $w_{i}=w\left(\hat{\tau}_{i}\right)=0$, for $i=1, \ldots, N$. Since, the equation $\hat{\mathbf{D}}_{:, 1: N}^{\alpha} w=0$ has no nonzero solution, the matrix $\hat{\mathbf{D}}_{:, 1: N}^{\alpha}$ is nonsingular.

REMARK 3.3. It is worth mentioning that the recently used classical pseudospectral matrices like PDMs $(\alpha=\mu=1)$ [31], FPIMs $(\mu=1)$ [35], and FPDMs $(\mu=1)$ [33] can be considered as special cases of our approach.

4. Equivalence of the generalized fractional pseudospectral schemes. The GFPDMs become extremely ill-conditioned when the number of collocation points is increased, and then the numerical solutions suffer from severe round-off errors. In this section, we generalize the fractional Birkhoff interpolation problem to generate efficient fractional integration preconditioning matrices for the ill-conditioned systems. Actually, we provide a stable way to compute the inverse of $\hat{\mathbf{D}}_{:, 1: N}^{\alpha}$, and then we show the equivalence between the integral and differential fractional pseudospectral schemes.

Let $\left\{\hat{\tau}_{i}\right\}_{i=1}^{N}$ be the JG points as before with $0=\hat{\tau}_{0}<\hat{\tau}_{1}<\ldots<\hat{\tau}_{N+1}=1$. For a function $u(\tau) \in C[0, T]$ satisfying ${ }_{0}^{c} D_{\tau}^{\alpha} u \in C(0, T]$, the generalization of the Caputo fractional Birkhoff interpolation problem is to find $h(\tau) \in \mathbf{M}_{N, \alpha}$ such that

$$
{ }_{0}^{c} D_{\hat{\tau}_{k}}^{\alpha} h(\tau)={ }_{0}^{c} D_{\hat{\tau}_{k}}^{\alpha} u(\tau), \quad k=1, \ldots, N, \quad h(0)=u(0) .
$$

The Birkhoff interpolation function $h(\tau)$ of $u(\tau)$ can be uniquely expressed as

$$
h(\tau)=u(0) \hat{\mathcal{B}}_{0}^{\alpha}(\tau)+\sum_{i=1}^{N} \hat{\mathcal{B}}_{i}^{\alpha}(\tau){ }_{0}^{c} D_{\hat{\tau}_{i}}^{\alpha} u(\tau),
$$

where $\left\{\hat{\mathcal{B}}_{i}^{\alpha} \in \mathbf{M}_{N, \alpha}\right\}_{i=0}^{N}$ are a generalization of the Birkhoff interpolation functions such that

$$
\begin{array}{lll}
\hat{\mathcal{B}}_{0}^{\alpha}(0)=1, & { }_{0}^{c} D_{\hat{\tau}_{k}}^{\alpha} \hat{\mathcal{B}}_{0}^{\alpha}(\tau)=0, & k=1, \ldots, N, \\
\hat{\mathcal{B}}_{i}^{\alpha}(0)=0, & { }_{0}^{c} D_{\hat{\tau}_{k}}^{\alpha} \hat{\mathcal{B}}_{i}^{\alpha}(\tau)=\delta_{k i}, & k, i=1, \ldots, N .
\end{array}
$$

Using (4.1) and the fact that ${ }_{0}^{c} D_{\tau}^{\alpha} \hat{\mathcal{B}}_{i}^{\alpha}(\tau) \in \mathbf{M}_{N-1, \alpha}$, we immediately obtain that $\hat{\mathcal{B}}_{0}^{\alpha}(\tau)=1$ and $\partial_{\tau}^{n} \hat{\mathcal{B}}_{i}^{\alpha}(0)=0$, with $1 \leq i \leq N$ and $n=0,1, \ldots, m-1$. Furthermore, since $\hat{h}_{j}^{\alpha} \in \mathbf{M}_{N-1, \alpha}$, we may write

$$
{ }_{0}^{c} D_{\tau}^{\alpha} \hat{\mathcal{B}}_{i}^{\alpha}(\tau)=\sum_{j=1}^{N} b_{j i} \hat{h}_{j}^{\alpha}(\tau)
$$


Taking $\tau=\hat{\tau}_{k}$, using the fact that $\hat{h}_{j}^{\alpha}\left(\tau_{k}\right)=\delta_{k j}$, and (4.2), we have $b_{k i}=\delta_{k i}$ and ${ }_{0}^{c} D_{\tau}^{\alpha} \hat{\mathcal{B}}_{i}^{\alpha}(\tau)=\hat{h}_{i}^{\alpha}(\tau)$. Hence, by applying the left RLFI operator, we obtain

$$
\hat{\mathcal{B}}_{i}^{\alpha}(\tau)=\sum_{n=0}^{m-1} \frac{\partial_{\tau}^{n} \hat{\mathcal{B}}_{i}^{\alpha}(0)}{n !} \tau^{n}+{ }_{0} I_{\tau}^{\alpha} \hat{h}_{i}^{\alpha}(\tau)={ }_{0} I_{\tau}^{\alpha} \hat{h}_{i}^{\alpha}(\tau), \quad i=1, \ldots, N .
$$

Moreover, taking $\tau=\hat{\tau}_{k}$, we have

$$
\hat{\mathcal{B}}_{i}^{\alpha}\left(\hat{\tau}_{k}\right)={ }_{0} I_{\hat{\tau}_{k}}^{\alpha} \hat{h}_{i}^{\alpha}(\tau)=\hat{\mathbf{I}}_{k i}^{\alpha, \alpha}, \quad k, i=1, \ldots, N .
$$

The following theorem show the equivalence of the proposed fractional pseudospectral schemes.

THEOREM 4.1. Let $\mathbf{I}$ be the identity matrix of order $N$. Then

$$
\hat{\mathbf{D}}_{:, 1: N}^{\alpha} \hat{\mathbf{I}}_{1: N,:}^{\alpha, \alpha}=\mathbf{I}
$$

where $\hat{\mathbf{I}}_{1: N,:}^{\alpha, \alpha}$ and $\hat{\mathbf{D}}_{:, 1: N}^{\alpha}$ are the matrices obtained by deleting the last row of $\hat{\mathbf{I}}^{\alpha, \alpha}$ and the first column of $\hat{\mathbf{D}}^{\alpha}$, respectively.

Proof. From (3.5) and (4.3), we have

$$
\hat{\mathcal{B}}_{i}^{\alpha}(\tau)=\sum_{j=1}^{N} \hat{l}_{j}^{\alpha}(\tau) \hat{\mathcal{B}}_{i}^{\alpha}\left(\hat{\tau}_{j}\right)=\sum_{j=1}^{N} \hat{l}_{j}^{\alpha}(\tau) \hat{\mathbf{I}}_{j i}^{\alpha, \alpha} .
$$

Applying the left CFD operator to (4.4) and evaluating at the points $\tau=\hat{\tau}_{k}$, with $1 \leq k \leq N$, yields

$$
{ }_{0}^{c} D_{\hat{\tau}_{k}}^{\alpha} \hat{\mathcal{B}}_{i}^{\alpha}(\tau)=\sum_{j=1}^{N}\left({ }_{0}^{c} D_{\hat{\tau}_{k}}^{\alpha} \hat{l}_{j}^{\alpha}(\tau)\right) \hat{\mathbf{I}}_{j i}^{\alpha, \alpha}
$$

Using (4.2), we have

$$
\sum_{j=1}^{N} \hat{\mathbf{D}}_{k j}^{\alpha} \hat{\mathbf{I}}_{j i}^{\alpha, \alpha}=\delta_{k i}, \quad k, i=1, \ldots, N
$$

which implies that $\hat{\mathbf{D}}_{:, 1: N}^{\alpha, \alpha} \hat{\mathbf{I}}_{1: N,:}^{\alpha, \alpha}=\mathbf{I}$.

Here we considered the generalization of fractional Birkhoff interpolation for the JG points, which can be extended to the case of FJGR points similarly. In the same way as in Theorem 4.1, we have

$$
\check{\mathbf{D}}_{:, 1: N}^{\alpha} \check{\mathbf{I}}^{\alpha, \alpha}=\mathbf{I} .
$$

\section{Fractional spectral collocation methods.}

5.1. The generalized integral fractional pseudospectral scheme. In this section, we establish a generalized integral fractional pseudospectral scheme with collocation at the JG and FJGR points for numerically solving initial value problems for FDEs. Consider the following general model of an FDE of order $\alpha \in(m-1, m]$ with $m \in \mathbb{N}$,

$$
\begin{aligned}
{ }_{0}^{c} D_{t}^{\alpha} u(t) & =f(t, u(t)), \quad t \in\left[0, t_{f}\right], \\
u^{(k)}(0) & =u_{k}, \quad k=0,1, \ldots, m-1,
\end{aligned}
$$


where $f$ is a nonlinear continuous function on $\left\{(t, u) \mid t \in\left[0, t_{f}\right], u \in \mathbb{R}\right\}$. Using (2.1), the above FDE can be rewritten in integral form (FIE) as

$$
u(t)=\varphi(t)+{ }_{0} I_{t}^{\alpha} f(t, u(t)),
$$

where $\varphi(t)=\sum_{i=0}^{m-1} \frac{u_{k}}{k !} t^{k}$.

5.1.1. Collocation at the JG points. The problem (5.2) can be discretized using JG collocation points $\left\{\hat{\tau}_{k}\right\}_{k=1}^{N}$ such that

$$
\hat{u}_{k}=\hat{\varphi}_{k}+{ }_{0} I_{\hat{\tau}_{k}}^{\alpha} f(t, u(t))=\hat{\varphi}_{k}+\sum_{i=1}^{N} \hat{\mathbf{I}}_{k i}^{\alpha, \mu} f\left(\hat{\tau}_{i}, \hat{u}_{i}\right), \quad k=1, \ldots, N,
$$

where $\left\{\hat{u}_{k} \approx u\left(\hat{\tau}_{k}\right), \hat{\varphi}_{k}=\varphi\left(\hat{\tau}_{k}\right)\right\}_{k=1}^{N}$. The matrix form of the above equation is

$$
\hat{u}=\hat{\varphi}+\hat{\mathbf{I}}_{1: N,:}^{\alpha, \mu} \hat{f},
$$

where $\hat{u}=\left(\hat{u}_{1}, \ldots, \hat{u}_{N}\right)^{T}, \hat{\varphi}=\left(\hat{\varphi}_{1}, \ldots, \hat{\varphi}_{N}\right)^{T}$, and $\hat{f}=\left(f\left(\hat{\tau}_{1}, \hat{u}_{1}\right), \ldots, f\left(\hat{\tau}_{N}, \hat{u}_{N}\right)\right)^{T}$. To gain more insight, without loss of generality, we suppose that

$$
f(t, u(t))=F(t)-\lambda(t) u(t)-g(t, u(t)) .
$$

We take the initial guess $\hat{u}^{1}=\hat{\varphi}$ and then use the following iterative method to solve the nonlinear FDEs:

$$
\left(\mathbf{I}+\hat{\mathbf{I}}_{1: N,:}^{\alpha, \mu} \hat{\Lambda}\right) \hat{u}^{i+1}=\hat{\varphi}+\hat{\mathbf{I}}_{1: N,:}^{\alpha, \mu} \hat{F}-\hat{\mathbf{I}}_{1: N,:}^{\alpha, \mu} \hat{g}^{i}, \quad i=1,2, \ldots,
$$

where

$$
\begin{aligned}
\hat{\Lambda} & =\operatorname{diag}\left(\lambda\left(\hat{\tau}_{1}\right), \ldots, \lambda\left(\hat{\tau}_{N}\right)\right)^{T}, \quad \hat{F}=\left(F\left(\hat{\tau}_{1}\right), \ldots, F\left(\hat{\tau}_{N}\right)\right)^{T}, \quad \text { and } \\
\hat{g}^{i} & =\left(g\left(\hat{\tau}_{1}, \hat{u}_{1}^{i}\right), \ldots, g\left(\hat{\tau}_{1}, \hat{u}_{N}^{i}\right)\right)^{T} .
\end{aligned}
$$

After evaluating $\left\{\hat{u}_{k}\right\}_{k=1}^{N}$, we can compute $\hat{u}_{N+1} \approx u\left(t_{f}\right)$ as

$$
\hat{u}_{N+1}=\varphi\left(t_{f}\right)+\sum_{i=1}^{N} \hat{\mathbf{I}}_{N+1, i}^{\alpha, \mu} f\left(\hat{\tau}_{i}, \hat{u}_{i}\right) .
$$

5.1.2. Collocation at the FJGR points. Using collocation at the FJGR points $\left\{\check{\tau}_{k}\right\}_{k=1}^{N}$ with the GFPIMs, the fractional integral equation (5.2) is discretized as

$$
\check{u}_{k}=\check{\varphi}_{k}+{ }_{0} I_{\check{\tau}_{k}}^{\alpha} f(t, u(t))=\check{\varphi}_{k}+\sum_{i=1}^{N} \check{\mathbf{I}}_{k i}^{\alpha, \mu} f\left(\check{\tau}_{i}, \check{u}_{i}\right), \quad k=1, \ldots, N,
$$

where $\left\{\check{u}_{k} \approx u\left(\check{\tau}_{k}\right), \check{\varphi}_{k}=\varphi\left(\check{\tau}_{k}\right)\right\}_{k=1}^{N}$. The matrix form of (5.3) can be written as

$$
\check{u}=\check{\varphi}+\check{\mathbf{I}}^{\alpha, \mu} \check{f},
$$

where $\check{u}=\left(\check{u}_{1}, \ldots, \check{u}_{N}\right)^{T}, \check{\varphi}=\left(\check{\varphi}_{1}, \ldots, \check{\varphi}_{N}\right)^{T}$, and $\check{f}=\left(f\left(\check{\tau}_{1}, \check{u}_{1}\right), \ldots, f\left(\check{\tau}_{1}, \check{u}_{N}\right)\right)^{T}$. Similar to the previous section, considering the initial guess $\check{u}^{1}=\check{\varphi}$, we have the following iterative scheme

$$
\left(\mathbf{I}+\check{\mathbf{I}}^{\alpha, \mu} \check{\Lambda}\right) \check{u}^{i+1}=\check{\varphi}+\check{\mathbf{I}}^{\alpha, \mu} \check{F}-\check{\mathbf{I}}^{\alpha, \mu} \check{g}^{i}, \quad i=1,2, \ldots,
$$

where

$$
\begin{aligned}
\check{\Lambda} & =\operatorname{diag}\left(\lambda\left(\check{\tau}_{1}\right), \ldots, \lambda\left(\check{\tau}_{N}\right)\right)^{T}, \quad \check{F}=\left(F\left(\check{\tau}_{1}\right), \ldots, F\left(\check{\tau}_{N}\right)\right)^{T}, \quad \text { and } \\
\check{g}^{i} & =\left(g\left(\check{\tau}_{1}, \check{u}_{1}^{i}\right), \ldots, g\left(\check{\tau}_{1}, \check{u}_{N}^{i}\right)\right)^{T} .
\end{aligned}
$$



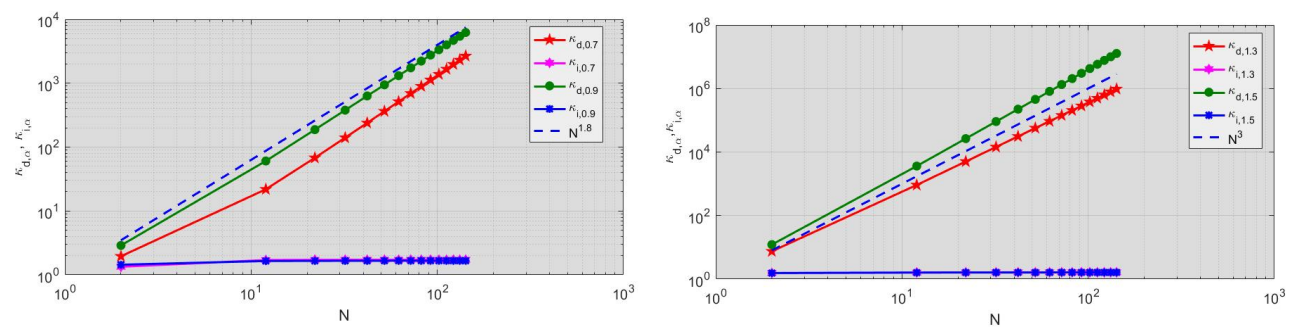

FIG. 5.1. The condition numbers $\kappa_{d, \alpha}$ and $\kappa_{i, \alpha}$ for some values of $\alpha$.

5.2. A well-conditioned generalized differential fractional pseudospectral scheme. In this section, we construct a well-conditioned fractional pseudospectral method for solving initial value problems for FDEs.

5.2.1. Collocation at the JG points. We consider the FDE (5.1) with $u^{(k)}(0)=0$, for $k=1, \ldots, m-1$, which can be discretized using collocation at the JG points $\left\{\hat{\tau}_{k}\right\}_{k=1}^{N}$ and $\hat{\tau}_{0}=0$ by

$$
\sum_{i=0}^{N} \hat{\mathbf{D}}_{k i}^{\alpha} \hat{u}_{i}=f\left(\hat{\tau}_{k}, \hat{u}_{k}\right), \quad k=1, \ldots, N
$$

In matrix form this reads

$$
\hat{\mathbf{D}}_{:, 1: N}^{\alpha} \hat{u}=-\hat{\mathbf{D}}_{:, 0}^{\alpha} \hat{u}_{0}+\hat{f} .
$$

Assume that $f(t, u(t))=F(t)-\lambda(t) u(t)-g(t, u(t))$. Then we can write

$$
\left(\hat{\mathbf{D}}_{:, 1: N}^{\alpha}+\hat{\Lambda}\right) \hat{u}=\hat{F}-\hat{\mathbf{D}}_{:, 0}^{\alpha} \hat{u}_{0}-\hat{g} .
$$

The present system is unpleasantly ill-conditioned. Hence, we construct a preconditioner for this system. Thanks to Theorem 4.1, we have

$$
\left(\mathbf{I}+\hat{\mathbf{I}}_{1: N,:}^{\alpha, \alpha} \hat{\Lambda}\right) \hat{u}=\hat{\mathbf{I}}_{1: N,:}^{\alpha, \alpha}\left(\hat{F}-\hat{\mathbf{D}}_{:, 0}^{\alpha} \hat{u}_{0}-\hat{g}\right) .
$$

Using the change of variable $\hat{u}:=\hat{\mathbf{I}}_{1: N,:}^{\alpha, \alpha} \hat{v}, \hat{v}=\left(\hat{v}_{1}, \ldots, \hat{v}_{N}\right)$, we obtain a well-conditioned preconditioned equation by

$$
\left(\mathbf{I}+\hat{\Lambda} \hat{\mathbf{I}}_{1: N,:)}^{\alpha, \alpha}\right) \hat{v}=\hat{F}-\hat{\mathbf{D}}_{:, 0}^{\alpha} \hat{u}_{0}-\hat{g}_{\hat{v}}
$$

where $\hat{g}_{\hat{v}}=\left(g\left(\hat{\tau}_{1}, \hat{\mathbf{I}}_{1,:}^{\alpha, \alpha} \hat{v}\right), \ldots, g\left(\hat{\tau}_{N}, \hat{\mathbf{I}}_{N,:}^{\alpha, \alpha} \hat{v}\right)\right)^{T}$. Also, we can choose the initial guess for $\hat{v}^{1}$ and define the following iterative scheme:

$$
\left(\mathbf{I}+\hat{\Lambda} \hat{\mathbf{I}}_{1: N,:}^{\alpha, \alpha}\right) \hat{v}^{i+1}=\hat{F}-\hat{\mathbf{D}}_{:, 0}^{\alpha} \hat{u}_{0}-\hat{g}_{v}^{i}, \quad i=1,2, \ldots
$$

In Figure 5.1, we compare the condition numbers $\kappa_{d, \alpha}$ and $\kappa_{i, \alpha}$ for the matrices $\hat{\mathbf{D}}_{:, 1: N}^{\alpha}+\mathbf{I}$ and $\mathbf{I}+\hat{\mathbf{I}}_{1: N,:}^{\alpha, \alpha}$, respectively, for some values of $\alpha$. It is observed that, as illustrated in Figure 5.1, the values of $\kappa_{d, \alpha}$ grow dramatically with an order of $O\left(N^{2 \alpha}\right)$, while the values of $\kappa_{i, \alpha}$ remain constant. Thus, the numerical solutions of the system (5.4) suffers from severe round-off errors in case of a large number of collocation points, while the system (5.5) is well-conditioned. 
5.2.2. Collocation at the FJGR points. The collocation scheme for FDEs (5.1) with $u^{(k)}(0)=0$, for $k=1, \ldots, m-1$, is

$$
\sum_{i=0}^{N} \check{\mathbf{D}}_{k i}^{\alpha} \check{u}_{i}=f\left(\check{\tau}_{k}, \check{u}_{k}\right), \quad k=1, \ldots, N
$$

and in matrix form,

$$
\check{\mathbf{D}}_{:, 1: N}^{\alpha} \check{u}=-\check{\mathbf{D}}_{:, 0}^{\alpha} \check{u}_{0}+\check{f} .
$$

Again, by setting $f(t, u(t))=F(t)-\lambda(t) u(t)-g(t, u(t))$ as in the previous section, we obtain

$$
\left(\mathbf{I}+\check{\Lambda}^{\alpha} \check{\mathbf{I}}^{\alpha, \alpha}\right) \check{v}^{i+1}=\check{F}-\check{\mathbf{D}}_{:, 0}^{\alpha} \check{u}_{0}-\check{g}_{\check{v}}^{i}, \quad i=1,2, \ldots,
$$

where $\check{u}:=\check{\mathbf{I}}_{1: N,:}^{\alpha, \alpha} \check{v}, \check{v}=\left(\check{v}_{1}, \ldots, \check{v}_{N}\right), \check{g}_{\check{v}}=\left(g\left(\check{\tau}_{1}, \check{\mathbf{I}}_{1,:}^{\alpha, \alpha} \check{u}_{1}\right), \ldots, g\left(\check{\tau}_{N}, \check{\mathbf{I}}_{N,:}^{\alpha, \alpha} \check{u}_{N}\right)\right)^{T}$.

REMARK 5.1. The proposed approaches can be developed for multi-term FDEs and variable-order FDEs, whose solutions can be either smooth or singular. For instance, we can generalize (5.1) to a multi-term linear or non-linear FDEs as

$$
\begin{aligned}
\sum_{i=1}^{k} \lambda_{i}(t)_{0}^{c} D_{t}^{\alpha_{i}} u(t) & =f(t, u), & & 0<\alpha_{1}<\alpha_{2}<\ldots<\alpha_{k}<m, \\
u^{(i)}(0) & =u_{i}, & i & =0,1, \ldots, m-1 .
\end{aligned}
$$

By defining $v(t):={ }_{0}^{c} D_{t}^{\alpha_{k}} u(t)$ and using (2.1), the above system can be put into the following form of an FIE:

$$
\sum_{i=1}^{k} \lambda_{i}(t){ }_{0} I_{t}^{\alpha_{k}-\alpha_{i}} v(t)+g(t)=f\left(t, \varphi(t)+{ }_{0} I_{t}^{\alpha_{k}} v(t)\right)
$$

where

$$
g(t)=\sum_{i=1}^{k} \sum_{j=\left\lceil\alpha_{i}\right\rceil}^{\left\lceil\alpha_{k}\right\rceil-1} \frac{u_{j} \lambda_{i}(t)}{\Gamma\left(j-\alpha_{i}+1\right)} t^{j-\alpha_{i}}
$$

\section{Convergence analysis.}

6.1. Error estimates. This section is devoted to the convergence analysis for the presented numerical methods. Also, we derive error estimates and convergence rates in the weighted $L^{2}$-norm. For this goal, let $\Omega=[0,1]$, and define the polynomial spaces and the Müntz spaces as

$$
\mathbf{P}_{N, \mu}(\Omega)=\operatorname{span}\left\{J_{k}^{0, \frac{1}{\mu}-1}(2 t-1)\right\}_{k=0}^{N}, \quad \mathbf{M}_{N, \mu}(\Omega)=\operatorname{span}\left\{J_{k}^{0, \frac{1}{\mu}-1}\left(2 \tau^{\mu}-1\right)\right\}_{k=0}^{N} .
$$

For any function $u \in C(\Omega)$, we define the generalized Lagrange interpolation operators $\hat{I}_{N}^{\mu} u$ and $\check{I}_{N}^{\mu} u$, satisfying

$$
\begin{aligned}
& \hat{I}_{N}^{\mu} u\left(\hat{\tau}_{i}\right):=\hat{I}_{N}^{0,-1+\frac{1}{\mu}} U\left(\frac{1+\hat{t}_{i}}{2}\right)=u\left(\hat{\tau}_{i}\right), \\
& \check{I}_{N}^{\mu} u\left(\check{\tau}_{i}\right):=\check{I}_{N}^{0,-1+\frac{1}{\mu}} U\left(\frac{1+\check{t}_{i}}{2}\right)=u\left(\check{\tau}_{i}\right),
\end{aligned}
$$


with $1 \leq i \leq N$. Recalling that $\hat{I}_{N}^{0,-1+\frac{1}{\mu}}$ and $\check{I}_{N}^{0,-1+\frac{1}{\mu}}$ are the Lagrange interpolation operators associated with the JG and FJGR points on $\Omega$, respectively, the pair of function $u(\tau)$ and $U(\tau)$ are related by $U(\tau):=u\left(\tau^{\frac{1}{\mu}}\right)$. On the other hand, we can write

$$
\hat{I}_{N}^{\mu} u(\tau)=\sum_{i=1}^{N} u\left(\hat{\tau}_{i}\right) \hat{h}^{\mu}(\tau), \quad \check{I}_{N}^{\mu} u(\tau)=\sum_{i=1}^{N} u\left(\check{\tau}_{i}\right) \check{h}^{\mu}(\tau) .
$$

In order to simplify the discussion and without loss of generality, in the following we consider the case of JG points. The same result also holds for the FJGR points. According to the Jacobi weight function $\omega^{\gamma, \eta}(t)=2^{\gamma+\eta}(1-t)^{\gamma} t^{\eta}, \gamma, \eta>-1$, we define a generalized weight function as $\tilde{\omega}^{a, b}(t)=2^{a+b}\left(1-t^{\mu}\right)^{a} t^{b \mu}$, with $a, b \in \mathbb{R}$. Thanks to [29], it is easy to obtain that

$$
\|u\|_{\tilde{\omega}^{0, b}}=\sqrt{c_{\mu}}\|U\|_{\omega^{0,-1+\frac{1}{\mu}+b}}, \quad\left\|\partial_{\tau} u\right\|_{\tilde{\omega}^{1,-1+\frac{2}{\mu}}}=\sqrt{\frac{1}{c_{\mu}}}\left\|\partial_{t} U\right\|_{\omega^{1, \frac{1}{\mu}}} .
$$

The key to express the error estimates is the introduction of the following differential operators,

$$
D_{\tau} u:=\frac{\partial U}{\partial t}=\frac{d \tau}{d t} \partial_{\tau} u, \quad D_{\tau}^{k} u:=\frac{\partial^{k} U}{\partial t^{k}}=\frac{d \tau}{d t} \partial_{\tau} D_{\tau}^{k-1} u, \quad k=2,3, \ldots,
$$

where $t=\tau^{\frac{1}{\mu}}$. Using the above notation, we obtain

$$
\left\|D_{\tau}^{k} u\right\|_{\tilde{\omega}^{k, k}}=\sqrt{c_{\mu}}\left\|\partial_{t}^{k} U\right\|_{\omega^{k, k-1+\frac{1}{\mu}}}, \quad k=0,1, \ldots
$$

To get the error estimates, analogous to the non-uniformly Jacobi-weighted Sobolev spaces $B_{0,-1+\frac{1}{\mu}}^{n}$ in [28],

$$
B_{0,-1+\frac{1}{\mu}}^{n}=\left\{U: \partial_{t}^{k} U \in L_{\omega^{k, k-1+\frac{1}{\mu}}}^{2}, \quad k=0,1, \ldots, n\right\},
$$

we define the following weighted Sobolev space

$$
\tilde{B}_{\mu}^{n}(\Omega)=\left\{u: D_{\tau}^{k} u \in L_{\tilde{\omega}^{k, k}}^{2}, \quad k=0,1, \ldots, n\right\}
$$

equipped with the norm and semi-norm

$$
\|u\|_{\tilde{B}_{\mu}^{n}}=\left(\sum_{k=0}^{n}\left\|D_{\tau}^{k} u\right\|_{\tilde{\omega}^{k, k}}^{2}\right)^{1 / 2}, \quad|u|_{\tilde{B}_{\mu}^{n}}=\left\|D_{\tau}^{n} u\right\|_{\tilde{\omega}^{k, k}} .
$$

Throughout this paper, we use the notation $A \preceq B$ to indicate that there exists a generic positive constant $c$, independent of $N$ and any function, such that $A \leq c B$. The following results from [28] show stability of the JG and FJGR interpolation operators. The error estimates in the next theorem for JG and FJGR interpolation can be found in [28].

THEOREM 6.1. For any function $U \in B_{0,-1+\frac{1}{\mu}}^{n}$, with $0 \leq l \leq n \leq N$, $n \geq 1$, we have

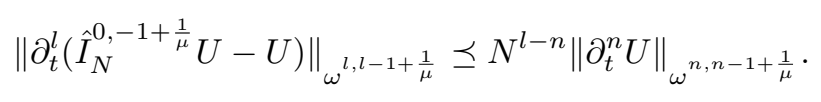

The same result holds for $\check{I}_{N}^{0,-1+\frac{1}{\mu}}$. 
From Theorem 6.1, an optimal error estimate for the generalized interpolation based on the JG points is obtained by

$$
\left\|D_{\tau}^{l}\left(\hat{I}_{N}^{\mu} u-u\right)\right\|_{\hat{\omega}^{l, l}} \preceq N^{l-n}\left\|D_{\tau}^{n} u\right\|_{\tilde{\omega}^{n, n}}, \quad u \in \tilde{B}_{\mu}^{n}(\Omega) .
$$

In the sequel, we present a convergence analysis for the generalized integral fractional pseudospectral scheme with collocation at the JG points in the $L^{2}$-norm. The same results also hold for collocation at the FJGR points. Without loss of generality, we consider the nonlinear FIE

$$
u(\tau)=F(\tau)+{ }_{0} I_{\tau}^{\alpha} \lambda(\tau) u(\tau)+{ }_{0} I_{\tau}^{\alpha} g(\tau, u(\tau)),
$$

where $g(t, u(t))$ satisfies a Lipschitz condition with Lipschitz constant $\gamma$,

$$
\left\|g\left(., u_{2}\right)-g\left(., u_{1}\right)\right\|_{L^{2}} \leq \gamma\left\|u_{2}-u_{1}\right\|_{L^{2}} .
$$

Let $u_{N}(\tau)=\sum_{i=1}^{N} \hat{u}_{i} \hat{h}_{i}^{\mu}(\tau)$ be the obtained numerical solution using the introduced scheme. In the following theorem, we give an error estimate for the numerical solution $u_{N}$ of the problem (6.3) in the sense of the $L^{2}$-norm.

THEOREM 6.2. Let $u$ be the exact solution of the fractional equation (6.3), and let the approximated solution $u_{N_{\tilde{B}}}$ be given by the scheme introduced in Section 5.1 with the JG collocation points. If $u \in \tilde{B}_{\mu}^{n}(\Omega)$, then the approximated solution $u_{N}$ converges to the exact solution $u$. Also, for sufficiently large $N$, the following error estimate holds:

$$
\left\|u-u_{N}\right\|_{L^{2}} \preceq N^{-n}\left\|D_{\tau}^{n} u\right\|_{\tilde{\omega}^{n, n}} .
$$

Proof. We observe that the solution $u$ of (6.3) satisfies

$$
u\left(\hat{\tau}_{k}\right)=F\left(\hat{\tau}_{k}\right)+{ }_{0} I_{\hat{\tau}_{k}}^{\alpha} \lambda(\tau) u(\tau)+{ }_{0} I_{\hat{\tau}_{k}}^{\alpha} g(\tau, u(\tau)) .
$$

It follows from the generalized integral fractional pseudospectral scheme that

$$
\hat{u}_{k}=F\left(\hat{\tau}_{k}\right)+\sum_{i=1}^{N} \hat{\mathbf{I}}_{k i}^{\alpha, \mu} \lambda\left(\hat{\tau}_{i}\right) \hat{u}_{i}+\sum_{i=1}^{N} \hat{\mathbf{I}}_{k i}^{\alpha, \mu} g\left(\hat{\tau}_{i}, \hat{u}_{i}\right) .
$$

Subtracting (6.6) from (6.5), we get

$$
\begin{gathered}
u\left(\hat{\tau}_{k}\right)-\hat{u}_{k}={ }_{0} I_{\hat{\tau}_{k}}^{\alpha} \lambda(\tau) u(\tau)-\sum_{i=1}^{N} \hat{\mathbf{I}}_{k i}^{\alpha, \mu} \lambda\left(\hat{\tau}_{i}\right) \hat{u}_{i}+{ }_{0} I_{\hat{\tau}_{k}}^{\alpha} g(\tau, u(\tau))-\sum_{i=1}^{N} \hat{\mathbf{I}}_{k i}^{\alpha, \mu} g\left(\hat{\tau}_{i}, \hat{u}_{i}\right) \\
={ }_{0} I_{\hat{\tau}_{k}}^{\alpha} \lambda(t)\left(u(t)-u_{N}(\tau)\right)+{ }_{0} I_{\hat{\tau}_{k}}^{\alpha}\left(g(\tau, u(\tau))-g\left(\tau, u_{N}(\tau)\right)\right) \\
\quad+{ }_{0} I_{\hat{\tau}_{k}}^{\alpha} \lambda(\tau) u_{N}(\tau)-\sum_{i=1}^{N} \hat{\mathbf{I}}_{k i}^{\alpha, \mu} \lambda\left(\hat{\tau}_{i}\right) \hat{u}_{i} \\
\quad+{ }_{0} I_{\hat{\tau}_{k}}^{\alpha} g\left(\tau, u_{N}(\tau)\right)-\sum_{i=1}^{N} \hat{\mathbf{I}}_{k i}^{\alpha, \mu} g\left(\hat{\tau}_{i}, \hat{u}_{i}\right) \\
:={ }_{0} I_{\hat{\tau}_{k}}^{\alpha} \lambda(\tau) e(\tau)+{ }_{0} I_{\hat{\tau}_{k}}^{\alpha} e_{g}(\tau)+\zeta_{k, 1}+\zeta_{k, 2},
\end{gathered}
$$

with

$$
\begin{aligned}
e(\tau) & =u(\tau)-u_{N}(\tau), & e_{g}(\tau) & =g(\tau, u(\tau))-g\left(\tau, u_{N}(\tau)\right), \\
\zeta_{k, 1} & ={ }_{0} I_{\hat{\tau}_{k}}^{\alpha} \lambda(\tau) u_{N}(\tau)-\sum_{i=1}^{N} \hat{\mathbf{I}}_{k i}^{\alpha, \mu} \lambda\left(\hat{\tau}_{i}\right) \hat{u}_{i}, & \zeta_{k, 2} & ={ }_{0} I_{\hat{\tau}_{k}}^{\alpha} g\left(\tau, u_{N}(\tau)\right)-\sum_{i=1}^{N} \hat{\mathbf{I}}_{k i}^{\alpha, \mu} g\left(\hat{\tau}_{i}, \hat{u}_{i}\right) .
\end{aligned}
$$


In $\zeta_{k, 1}$ and $\zeta_{k, 2}$, the error term is $\epsilon_{k i}^{\mu}={ }_{0} I_{\hat{\tau}_{k}}^{\alpha} \hat{h}_{i}^{\mu}-\hat{\mathbf{I}}_{k i}^{\alpha, \mu}$. Clearly, $\epsilon_{k i}^{\mu}$, with $1 \leq i, k \leq N$, can be bounded by the estimates for the integration errors produced by JG-type quadrature formulas relative to the Jacobi weight $\omega^{\alpha-1, \mu}$ (see (3.2)). Using [7, Lemma 3.3], we have $\left\|\epsilon_{k i}^{\mu}\right\| \preceq M^{-n}\left\|\partial_{\tau}^{n} \hat{h}_{i}^{\mu}\right\|_{\omega^{\alpha-1+n, \mu+n}}$ for some $n \geq 1$. Then, for $\frac{n-1}{3} \leq \mu<\frac{n}{3}$, we obtain $\left\|\epsilon_{k i}^{\mu}\right\|=O\left(M^{-n}\right)$. The numerical results show that this convergence rate is rather pessimistic. Using discussions in [14] and Remark 3.1, we have the optimal rate of convergence for the integration error $\epsilon_{k i}^{\mu}$ given as $\left.\left\|\epsilon_{k i}^{\mu}\right\|=O\left(M^{-2\left(1+\left(2+l_{\mu}\right) \mu\right.}\right)\right)$. The numerical results show that this rate is much closer to the numerical rate. Considering the proper parameter $l_{\mu}$ and taking $M$ sufficiently large compared to the value of $N$, we can reduce the effect of the integral error as much as possible. According to the above discussions, the dominant error in the error equation (6.7) will be in the remaining terms, therefore, we exclude the errors in $\zeta_{k, 1}$ and $\zeta_{k, 2}$. Multiplying $\hat{h}_{k}^{\mu}(\tau)$ on both side of the error equation (6.7) and summing up from $k=1$ to $k=N$ yields

$$
\hat{I}_{N}^{\mu} u(\tau)-u_{N}(\tau) \approx \sum_{k=1}^{N}\left({ }_{0} I_{\hat{\tau}_{k}}^{\alpha} \lambda(\tau) e(\tau)\right) \hat{h}_{k}^{\mu}(\tau)+\sum_{k=1}^{N}\left({ }_{0} I_{\hat{\tau}_{k}}^{\alpha} e_{g}(\tau)\right) \hat{h}_{k}^{\mu}(\tau) .
$$

Then, we have

$$
\begin{aligned}
u_{N}(\tau) & \left.\approx \hat{I}_{N}^{\mu} u(\tau)-\sum_{k=1}^{N}\left({ }_{0} I_{\hat{\tau}_{k}}^{\alpha} \lambda(\tau) e(\tau)\right) \hat{h}_{k}^{\mu}(\tau)-\sum_{k=1}^{N}{ }_{0} I_{\hat{\tau}_{k}}^{\alpha} e_{g}(\tau)\right) \hat{h}_{k}^{\mu}(\tau) \\
& =\hat{I}_{N}^{\mu} u(\tau)-\hat{I}_{N}^{\mu}\left({ }_{0} I_{\tau}^{\alpha} \lambda(\tau) e(\tau)\right)-\hat{I}_{N}^{\mu}\left({ }_{0} I_{\tau}^{\alpha} e_{g}(\tau)\right) .
\end{aligned}
$$

Consequently, from (6.8), we get

$$
\begin{gathered}
e(\tau) \approx u(\tau)-\hat{I}_{N}^{\mu} u(\tau)+\hat{I}_{N}^{\mu}\left({ }_{0} I_{\tau}^{\alpha} \lambda(\tau) e(\tau)\right)-{ }_{0} I_{\tau}^{\alpha} \lambda(\tau) e(\tau)+{ }_{0} I_{\tau}^{\alpha} \lambda(\tau) e(\tau) \\
+\hat{I}_{N}^{\mu}\left({ }_{0} I_{\tau}^{\alpha} e_{g}(\tau)\right)-{ }_{0} I_{\tau}^{\alpha} e_{g}(\tau)+{ }_{0} I_{\tau}^{\alpha} e_{g}(\tau) \\
={ }_{0} I_{\tau}^{\alpha} \lambda(\tau) e(\tau)+{ }_{0} I_{\tau}^{\alpha} e_{g}(\tau)+\zeta^{1}+\zeta^{2}+\zeta^{3}
\end{gathered}
$$

where

$$
\begin{aligned}
& \zeta^{1}=u(\tau)-\hat{I}_{N}^{\mu} u(\tau), \quad \zeta^{2}=\hat{I}_{N}^{\mu}\left({ }_{0} I_{\tau}^{\alpha} \lambda(\tau) e(\tau)\right)-{ }_{0} I_{\tau}^{\alpha} \lambda(\tau) e(\tau), \quad \text { and } \\
& \zeta^{3}=\hat{I}_{N}^{\mu}\left({ }_{0} I_{\tau}^{\alpha} e_{g}(\tau)\right)-{ }_{0} I_{\tau}^{\alpha} e_{g}(\tau) .
\end{aligned}
$$

Taking the $L^{2}$-norm in (6.9), we have

$$
\|e\|_{L^{2}} \leq\left\|_{0} I_{\tau}^{\alpha} \lambda(\tau) e(\tau)\right\|_{L^{2}}+\left\|{ }_{0} I_{\tau}^{\alpha} e_{g}(\tau)\right\|_{L^{2}}+\left\|\zeta^{1}\right\|_{L^{2}}+\left\|\zeta^{2}\right\|_{L^{2}}+\left\|\zeta^{3}\right\|_{L^{2}}
$$

Since for $\alpha>0,{ }_{0} I_{\tau}^{\alpha}: L^{2}(\Omega) \longrightarrow L^{2}(\Omega)$ is a bounded linear operator (see [6]), we have

$$
\left\|I_{\tau} I_{\tau}^{\alpha} \lambda(\tau) e(\tau)\right\|_{L^{2}} \leq C_{1}\|\lambda e\|_{L^{2}} \leq C_{1} \max _{\tau \in(0,1)}|\lambda(\tau)|\|e\|_{L^{2}}
$$

Using the Lipschitz condition, we obtain $\left\|_{0} I_{\tau}^{\alpha} e_{g}(\tau)\right\|_{L^{2}} \leq C_{2} \gamma\|e\|_{L^{2}}$ for some constants $C_{i}$. Taking $l=0$ in (6.2), we have $\left\|\zeta^{1}\right\|_{L^{2}} \preceq N^{-n}\left\|D_{\tau}^{n} u\right\|_{\tilde{\omega}^{n, n}}$. From $\tilde{B}_{\mu}^{0}(\Omega) \subseteq \tilde{B}_{\mu}^{n}(\Omega)$, we get

$$
\left\|\zeta^{2}\right\|_{L^{2}} \leq C_{3}\left\|_{0} I_{\tau}^{\alpha} \lambda(\tau) e(\tau)\right\|_{L^{2}} \leq C_{1} C_{3} \max _{\tau \in(0,1)}|\lambda(\tau)|\|e\|_{L^{2}}
$$

A similar analysis can be performed for $\zeta^{3}$. Thus, we have

$$
\left\|\zeta^{3}\right\|_{L^{2}} \leq C_{4}\left\|_{0} I_{\tau}^{\alpha} e_{g}(\tau)\right\|_{L^{2}} \leq C_{2} C_{4} \gamma\|e\|_{L^{2}} .
$$

These estimates show that the numerical solution $u_{N}(\tau)$ converges to the exact solution, and also the desired estimate (6.4) is obtained. 


\section{ETNA}

Kent State University and

Johann Radon Institute (RICAM)

6.2. Convergence rates. At the end of this section, we theoretically provide accurate convergence rates for our numerical methods, which will be justified using numerical examples. We express the convergence rates as a function of $\alpha, \mu$, and the singularity index of the solution. We first present the convergence rate for the error of the generalized interpolation operator. For this objective, assume that the singularity of $u(\tau) \in L_{\tilde{\omega}^{0, b}}^{2}$ near $t=0$ behaves like $\sum_{i=1}^{\infty} O\left(t^{\delta_{i}}\right)$, where $0<\delta_{1}<\delta_{2}<\ldots$ Then the singularity of $U(\tau)=u\left(\tau^{\frac{1}{\mu}}\right)$ behaves like $\sum_{i=1}^{\infty} O\left(t^{\frac{\delta_{i}}{\mu}}\right)$. Inspired by [16] and using (6.1), we can derive the convergence rate of the interpolation error as

$$
\left\|u-\hat{I}_{N}^{\mu} u\right\|_{\tilde{\omega}^{0, b}}=\sqrt{c_{\mu}}\left\|U-\hat{I}_{N}^{0,-1+\frac{1}{\mu}} U\right\|_{\omega^{0,-1+\frac{1}{\mu}+b}} \simeq O\left(N^{-R_{\mu, b}}\right),
$$

where, if $\alpha \mid \delta_{j}$, for $j=1,2, \ldots, i-1$, and $\alpha \nmid \delta_{i}$, we have for the rate exponent that $R_{\mu, b}=1+2 \frac{\delta_{i}}{\mu}+\frac{1}{\mu}+b-1=\frac{1+2 \delta_{i}}{\mu}+b$. Moreover, if $\alpha \mid \delta_{j}, j=1,2, \ldots$, then exponential convergence is observed.

To obtain the convergence rate of the proposed schemes, we consider the model fractional equation ${ }_{0}^{c} D_{t}^{\alpha} u(t)=f(t)$ with initial conditions $u^{(k)}(0)=u_{k}$, for $k=0,1, \ldots, m-1$. Let $u_{N}(\tau)=\sum_{i=1}^{N} \hat{u}_{i} \hat{h}_{i}^{\mu}(\tau)$ be the approximate solution obtained by the proposed numerical schemes. To reach a high rate of convergence and exponential convergence, the index $\mu$ must be chosen such that it balances the singularity of the solution $u$ and the singularity of the forcing term $f$. Considering the stability of the method, we try to choose $\mu$ such that $\mu \mid \delta_{i}$ and $\mu \mid \delta_{j}-\alpha$ for some index of $i, j=1,2, \ldots$ By this, we obtain a convergence rate for the error estimates as $\left\|u-u_{N}\right\|_{L^{2}(I)} \simeq O\left(N^{-r_{\mu, \alpha}}\right)$. Motivated and inspired by the first Strang Lemma (see [28]) and using $\left(u-u_{N}\right)^{(k)}(0)=0$, for $k=0,1, \ldots, m-1$, we use the following trivial inequality

$$
\left\|u-u_{N}\right\|_{L^{2}} \leq\left\|u-\hat{I}_{N}^{\mu} u\right\|_{L^{2}}+\|{ }_{0} I_{t}^{\alpha}\left({ }_{0}^{c} D_{t}^{\alpha}\left(u-u_{N}\right) \|_{L^{2}} .\right.
$$

By the generalized Hardy's inequality (see [7]), there exists a constant $c_{1}$ independent of $N$ and $u$ such that

$$
\| I_{0} I_{t}^{\alpha}\left({ }_{0}^{c} D_{t}^{\alpha}\left(u-u_{N}\right)\left\|_{L^{2}} \leq c_{1}\right\|_{0}^{c} D_{t}^{\alpha}\left(u-u_{N}\right) \|_{\omega^{0, \kappa}},\right.
$$

where $\kappa$ must be chosen near 1 such that

$$
\sup _{-1<x<1} \int_{-1}^{x}(1+t)^{-\kappa} d t<\infty
$$

Then, we get

$$
\begin{aligned}
\left\|u-u_{N}\right\|_{L^{2}} & \leq\left\|u-\hat{I}_{N}^{\mu} u\right\|_{L^{2}}+\left\|_{0}^{c} D_{t}^{\alpha}\left(u-u_{N}\right)\right\|_{\omega^{0, \kappa}} \\
& \preceq\left\|u-\hat{I}_{N}^{\mu} u\right\|_{L^{2}}+\left\|f-\bar{I}_{N}^{\mu} f\right\|_{\omega^{0, \kappa}},
\end{aligned}
$$

where $\bar{I}_{N}^{\mu} u(\tau):=\tau^{\alpha} \hat{I}_{N}^{\mu}\left(\tau^{-\alpha} u(\tau)\right)$. In the inequality in (6.11), the term $\left\|u-\hat{I}_{N}^{\mu} u\right\|_{L^{2}(I)}$ is related to $u(t) \sim t^{\delta_{1}}+t^{\delta_{2}}+\ldots$ and $\left\|f-\bar{I}_{N}^{\mu} f\right\|_{\omega^{0, \kappa}}$ is related to the forcing term $f(t) \sim t^{\delta_{1}-\alpha}+t^{\delta_{2}-\alpha}+\ldots$ For the first term, using (6.10), we have

$$
\left\|u-\hat{I}_{N}^{\mu} u\right\|_{L^{2}(I)}=O\left(N^{\frac{-1}{\mu}(1+2 \hat{\delta})}\right) .
$$

For the second term, since $u_{N} \in \mathbf{M}_{N-1, \mu}$ it follows that ${ }_{0}^{c} D_{t}^{\alpha} u_{N} \in t^{-\alpha} \mathbf{M}_{N-1, \mu}$. Thus, we get

$$
\begin{aligned}
\left\|f-\bar{I}_{N}^{\mu} f\right\|_{\omega^{0, \kappa}} & =\left\|f-t^{\alpha} \hat{I}_{N}^{\mu} t^{-\alpha} f\right\|_{\omega^{0, \kappa}}=\left\|t^{\alpha}\left(t^{-\alpha} f-\hat{I}_{N}^{\mu} t^{-\alpha} f\right)\right\|_{\omega^{0, \kappa}} \\
& =\left\|t^{-\alpha} f-\hat{I}_{N}^{\mu} t^{-\alpha} f\right\|_{\omega^{0, \kappa+2 \alpha}} .
\end{aligned}
$$


TABLE 7.1

Numerical rates and the approximation errors when $\left(\alpha, \mu, \delta_{1}, \delta_{2}\right)=(0.8,0.8,0.8,1)$ for different $N$.

\begin{tabular}{lllllll}
$N$ & $R_{\mu, 0}$ & $r_{\mu, \alpha}$ & $\hat{r}_{\mu, \alpha}$ & $\left\|u-\hat{I}_{N}^{\mu} u\right\|_{L^{2}}$ & $\left\|u-u_{N}\right\|_{L^{2}}$ & $\|\mathbf{u}-\tilde{\mathbf{u}}\|_{\infty}$ \\
\hline 10 & 3.9793 & 3.2333 & 2.6044 & $4.1219 \mathrm{e}-05$ & $8.6588 \mathrm{e}-05$ & $3.6078 \mathrm{e}-04$ \\
20 & 3.8444 & 3.1035 & 2.5498 & $3.2930 \mathrm{e}-06$ & $1.2800 \mathrm{e}-05$ & $7.5963 \mathrm{e}-05$ \\
50 & 3.7822 & 3.0362 & 2.5193 & $1.1221 \mathrm{e}-07$ & $9.1827 \mathrm{e}-07$ & $8.6086 \mathrm{e}-06$ \\
100 & 3.7650 & 3.0167 & 2.5059 & $8.5208 \mathrm{e}-08$ & $1.1967 \mathrm{e}-07$ & $1.5828 \mathrm{e}-06$ \\
150 & 3.7548 & 3.0059 & 2.5063 & $1.8767 \mathrm{e}-09$ & $3.6006 \mathrm{e}-08$ & $5.8208 \mathrm{e}-07$
\end{tabular}

TABLE 7.2

Numerical rates and the approximation errors when $\left(\alpha, \mu, \delta_{1}, \delta_{2}\right)=(0.31,0.7,0.67,0.93)$ for different $N$.

\begin{tabular}{lllllll}
$N$ & $R_{\mu, 0}$ & $r_{\mu, \alpha}$ & $\hat{r}_{\mu, \alpha}$ & $\left\|u-\hat{I}_{N}^{\mu} u\right\|_{L^{2}}$ & $\left\|u-u_{N}\right\|_{L^{2}}$ & $\|\mathbf{u}-\tilde{\mathbf{u}}\|_{\infty}$ \\
\hline 10 & 4.9394 & 3.4232 & 2.0663 & $1.3690 \mathrm{e}-05$ & $8.8073 \mathrm{e}-05$ & $6.4988 \mathrm{e}-04$ \\
20 & 4.7746 & 3.3541 & 1.9870 & $5.9438 \mathrm{e}-07$ & $8.4383 \mathrm{e}-06$ & $1.9145 \mathrm{e}-04$ \\
50 & 4.7078 & 3.3366 & 1.9448 & $8.8998 \mathrm{e}-09$ & $4.7610 \mathrm{e}-07$ & $3.5420 \mathrm{e}-05$ \\
100 & 4.6985 & 3.3362 & 1.9310 & $1.0350 \mathrm{e}-10$ & $5.0301 \mathrm{e}-08$ & $9.6050 \mathrm{e}-06$ \\
150 & 4.6949 & 3.3398 & 1.9261 & $3.0297 \mathrm{e}-11$ & $1.3290 \mathrm{e}-08$ & $4.4507 \mathrm{e}-06$
\end{tabular}

Since the singularity index of $t^{-\alpha} f$ is $\delta_{j}-2 \alpha$, with $j=1,2, \ldots$, using (6.10), we obtain

$$
\left\|f-\bar{I}_{N}^{\mu} f\right\|_{\omega^{0, \kappa}}=O\left(N^{-\left(1+2 \frac{\check{\delta}-2 \alpha}{\mu}+\frac{2 \alpha+\kappa}{\mu}+\frac{1}{\mu}-1\right)}\right)=O\left(N^{-\left(\frac{2}{\mu}(\check{\delta}-\alpha)+\frac{\kappa+1}{\mu}\right)}\right) .
$$

From (6.12) and (6.13), we have that $r_{\mu, \alpha}=\min \left(\frac{1}{\mu}(1+2 \hat{\delta}), \frac{2}{\mu}(\check{\delta}-\alpha)+\frac{\kappa+1}{\mu}\right)$. More detailed, if $\mu \mid \alpha$ is related to ${ }_{0}^{c} D_{t}^{\alpha} u_{N}(t)=\sum_{i=1}^{N} \hat{u}_{i}\left({ }_{0}^{c} D_{t}^{\alpha} \hat{h}_{i}^{\mu}(\tau)\right)$, and $\mu \mid \delta_{i}$, with $i=1, \ldots, j-1$, and $\mu \nmid \delta_{j}$ is related to $u(t) \sim t^{\delta_{1}}+t^{\delta_{2}}+\ldots$, we have $\hat{\delta}=\delta_{j}$. Also, if $\mu \mid \delta_{i}-\alpha$, with $i=1, \ldots, k-1$, and $\mu \nmid \delta_{k}-\alpha$ is related to $f(t) \sim t^{\delta_{1}-\alpha}+t^{\delta_{2}-\alpha}+\ldots$, we have $\check{\delta}=\delta_{k}$. It is also noteworthy to point out that if we have $\mu \mid \delta_{i}$, with $i=1,2, \ldots$, and $\mu \mid \alpha$, then we get exponential convergence. For the numerical experiments, we define the convergence rate for the error as

$$
\left.\|\mathbf{u}-\tilde{\mathbf{u}}\|_{\infty}=\max _{1 \leq i \leq N} \mid u\left(\hat{\tau}_{i}\right)-\hat{u}_{i}\right) \mid=O\left(N^{-\hat{r}_{\mu, \alpha}}\right)
$$

where $\mathbf{u}=\left(u\left(\hat{\tau}_{1}\right), \ldots, u\left(\hat{\tau}_{N}\right)\right)^{T}$ and $\tilde{\mathbf{u}}=\left(\hat{u}_{1}, \ldots, \hat{u}_{N}\right)^{T}$. One can use the Sobolev inequality $\|\mathbf{u}-\tilde{\mathbf{u}}\|_{\infty} \preceq\left(\left\|u-u_{N}\right\|_{L^{2}}+\left\|\partial_{\tau}\left(u-u_{N}\right)\right\|_{L^{2}}\right)$ to estimate the numerical convergence rate as $\hat{r}_{\mu, \alpha}=\min \left(2 \frac{\hat{\delta}}{\mu}, \frac{2}{\mu}(\check{\delta}-\alpha)+\frac{\kappa+1}{\mu}\right)$.

7. Applications and numerical results. In this section, in order to test the efficiency and accuracy of the proposed schemes, we present some numerical examples. In the first example, we illustrate the theoretical convergence rates presented in this paper. In all examples, the $L^{2}$-norm is evaluated by JG quadrature with 200 points.

EXAMPLE 7.1. For a better treatment of the singularity, let $u(t)=t^{\delta_{1}}+t^{\delta_{2}}$ be the exact solution of ${ }_{0}^{c} D_{t}^{\alpha} u(t)=f(t)$ with $\alpha \leq \delta_{1}<\delta_{2}$. We investigate this example for various instances of the quadrupel parameter $\left(\alpha, \mu, \delta_{1}, \delta_{2}\right)$. The notation is such that the triplet $\left(R_{\mu, b}^{*}, r_{\mu, \alpha}^{*}, \hat{r}_{\mu, \alpha}^{*}\right)$ are the presented convergence rates in Section 7 and the triplet $\left(R_{\mu, b}, r_{\mu, \alpha}, \hat{r}_{\mu, \alpha}\right)$ are the numerical convergence rates.

In Table 7.1, we list the numerical convergence rates $\left(R_{\mu, 0}, r_{\mu, \alpha}, \hat{r}_{\mu, \alpha}\right)$, and the approximation errors $\left\|u-\hat{I}_{N}^{\mu} u\right\|_{L^{2}},\left\|u-u_{N}\right\|_{L^{2}}$, and $\|\mathbf{u}-\tilde{\mathbf{u}}\|_{\infty}$ for some values of the collocation points $N$ when $\left(\alpha, \mu, \delta_{1}, \delta_{2}\right)=(0.8,0.8,0.8,1)$. We observe that by increasing the 


\section{ETNA}

Kent State University and

Johann Radon Institute (RICAM)

TABLE 7.3

Numerical rates, our expected rates, and the approximation errors when $\left(\alpha, \mu, \delta_{1}, \delta_{2}\right)=(0.1, \mu, 0.1,0.2)$ with different $\mu$ and $N=70$.

\begin{tabular}{lllllll}
$\mu$ & $r_{\mu, \alpha}$ & $r_{\mu, \alpha}^{*}$ & $\hat{r}_{\mu, \alpha}$ & $\hat{r}_{\mu, \alpha}^{*}$ & $\left\|u-u_{N}\right\|_{L^{2}}$ & $\|\mathbf{u}-\tilde{\mathbf{u}}\|_{\infty}$ \\
\hline 0.3 & 4.0113 & 4.0000 & 1.3333 & 1.3256 & $4.2548 \mathrm{e}-7$ & $3.6541 \mathrm{e}-05$ \\
0.5 & 2.4943 & 2.4000 & 0.8017 & 0.8000 & $1.0581 \mathrm{e}-05$ & $2.0823 \mathrm{e}-04$ \\
0.7 & 1.8200 & 1.7143 & 0.5746 & 0.5714 & $1.3879 \mathrm{e}-04$ & $6.3628 \mathrm{e}-04$ \\
0.9 & 1.4299 & 1.3333 & 0.4473 & 0.444 & $5.2897 \mathrm{e}-04$ & $1.1052 \mathrm{e}-03$ \\
1 & 1.2925 & 1.2000 & 0.4028 & 0.4000 & $9.3568 \mathrm{e}-04$ & $1.5003 \mathrm{e}-03$
\end{tabular}

TABLE 7.4 $\left(\alpha, \delta_{1}, \delta_{2}\right)$

Our expected rates with $\mu=\alpha, 1$ and the introduced convergence rates in $[6,35,43]$ for several cases of

\begin{tabular}{llllll}
$\left(\alpha, \delta_{1}, \delta_{2}\right)$ & $r_{\alpha, \alpha}^{*}$ & $r_{1, \alpha}^{*}$ & {$[43]$} & {$[35]$} & {$[6]$} \\
\hline$(0.8,0.8,1.2)$ & 3.5 & 2.6 & 1.8 & 1 & - \\
$(0.4,0.5,0.7)$ & 5 & 2 & 1.4 & - & - \\
$(0.6,0.7,0.85)$ & 3.66 & 2.2 & 1.6 & 1 & - \\
$(0.1,0.2,0.3)$ & e. c & 1.4 & 1.1 & - & -
\end{tabular}

number of points, the numerical rates converge to our expected rates $\left(R_{\mu, 0}^{*}, r_{\mu, \alpha}^{*}, \hat{r}_{\mu, \alpha}^{*}\right)=$ $(3.75,3,2.5)$. In Table 7.2 , we provide similar results as in Table 7.1 for the parameter setting $\left(\alpha, \mu, \delta_{1}, \delta_{2}\right)=(0.31,0.7,0.67,0.93)$, where the numerical rates converge to $\left(R_{\mu, 0}^{*}, r_{\mu, \mu}^{*}, \hat{r}_{\mu, \mu}^{*}\right)=(4.68,3.3419,1.9143)$. It is observed that the desired rates are obtained, which strongly improve with increasing $N$.

In Table 7.3, for the case $\left(\alpha, \mu, \delta_{1}, \delta_{2}\right)=(0.1, \mu, 0.1,0.2)$, the numerical rates, our expected rates, and the errors $\left\|u-u_{N}\right\|_{L^{2}}$ and $\|\mathbf{u}-\tilde{\mathbf{u}}\|_{\infty}$ for some values of $\mu$ and $N=70$ are listed. As we can see, by decreasing $\mu$, exponential convergence (e. c) is achieved. In Table 7.4, we compare our convergence rates with $\mu=\alpha, 1$ and the rates in [6,35,43] for several cases of $\left(\alpha, \delta_{1}, \delta_{2}\right)$. We see that our expected rates of the proposed method are higher and more realistic than those in $[6,35,43]$.

In Figure 7.1 (left), the numerical rates $r_{\mu, \alpha}$ and our expected rates $r_{\mu, \alpha}^{*}$ are displayed versus $\delta_{2}$ for several cases of $\left(\alpha, \mu, \alpha, \delta_{2}\right)$. We observe that for $\delta_{2} \leq 2 \alpha-1$, we have $r_{\mu, \alpha}^{*}=\frac{2}{\mu}\left(1+\delta_{2}-\alpha\right)$, and for $\delta_{2} \geq 2 \alpha-1$, we have $r_{\mu, \alpha}^{*}=\frac{1}{\mu}\left(1+2 \delta_{1}\right)$. Also, similar observations for several cases of $\left(\alpha, \mu, \delta_{1}, \delta_{2}\right)$ with various parameter $\mu$ and $N=70$ can be observed in Figure 7.1 (right).

In Figure 7.2, we consider four cases of $\left(\alpha, \mu, \delta_{1}, \delta_{2}\right)$ given by

$\left(\alpha, \mu, \delta_{1}, \delta_{2}\right) \in\{(0.8,0.8,0.8,1.2),(0.4,0.7,0.5,0.7,(1.1,1.1,1.2,1.5),(0.1,1,0.2,0.3)\}$.

There, the errors $\left\|u-u_{N}\right\|_{L^{2}}$ and $N^{-r_{\mu, \alpha}^{*}}$ (left), and the errors $\left\|u-\hat{I}_{N}^{\mu} u\right\|_{L^{2}}$ and $N^{-R_{\mu, 0}^{*}}$ (right) versus the values of $N$ are at display. We observe that $\left\|u-u_{N}\right\|_{L^{2}}$ and $\left\|u-\hat{I}_{N}^{\mu} u\right\|_{L^{2}}$ decay with an order of $O\left(N^{-r_{\mu, \alpha}^{*}}\right)$ and $O\left(N^{-R_{\mu, 0}^{*}}\right)$, respectively, demonstrating the desired spectral rate of convergence. Again, we can see that our proposed rates are in accordance with the numerical results for nonsmooth solutions.

Finally, we use a parameter settings of $\left(\alpha, \mu, \delta_{1}, \delta_{2}\right)$ as

$$
\begin{aligned}
& \left(\alpha, \mu, \delta_{1}, \delta_{2}\right) \in\{(0.4,0.6,0.6,1), \quad(0.3,0.7,0.6,0.9), \quad(1.2,1,1.2,1.5), \\
& (0.9,0.9,1,1.5), \quad(1.1,1,1.1,1.5), \\
& (0.9,0.9,1,1.2), \quad(0.4,0.6,0.6,1),(1.3,1.5,1.4,1.7)\} \text {, }
\end{aligned}
$$



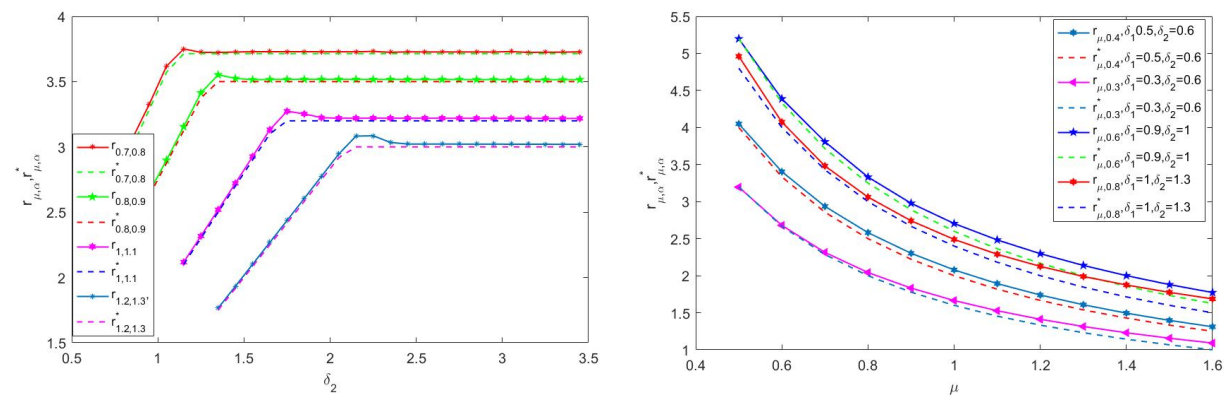

FIG. 7.1. The curves of the numerical rates $r_{\mu, \alpha}$ and our expected rates $r_{\mu, \alpha}^{*}$ plotted against different $\delta_{2}$ with $\alpha=\delta_{1}$ (left) and against different $\mu$ (right) for several cases of $\left(\alpha, \mu, \delta_{1}, \delta_{2}\right)$ when $N=70$.
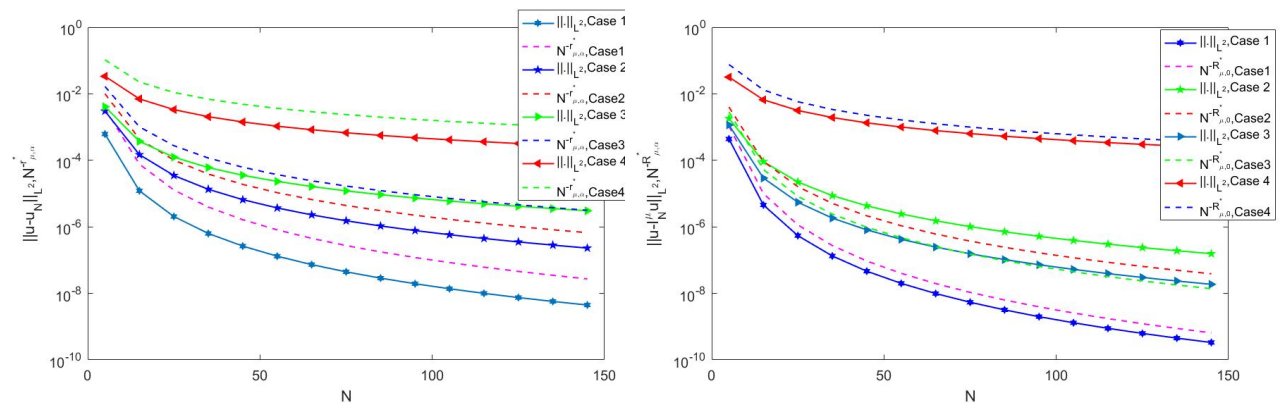

FIG. 7.2. The behavior of $\left\|u-u_{N}\right\|_{L^{2}}, N^{-r_{\mu, \alpha}^{*}},\left\|u-\hat{I}_{N}^{\mu} u\right\|_{L^{2}}$, and $N^{-R_{\mu, 0}^{*}}$ versus the values of $N$ for several cases of $\left(\alpha, \mu, \delta_{1}, \delta_{2}\right)$.
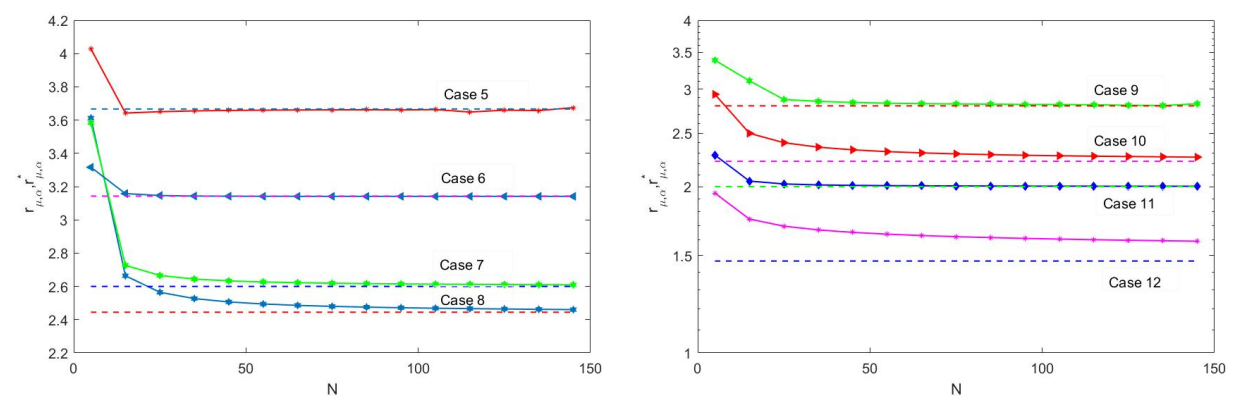

FIG. 7.3. The graph of the numerical rates $r_{\mu, \alpha}$ and our expected rates $r_{\mu, \alpha}^{*}$ versus $N$ for Cases 5-12 of $\left(\alpha, \mu, \delta_{1}, \delta_{2}\right)$.

TABLE 7.5

$L^{\infty}$-norm error and CPU time using MATLAB on a $2.60 \mathrm{GHz}$ personal computer.

\begin{tabular}{l|ll|ll|ll|ll} 
& \multicolumn{2}{|c|}{ FSCM [40] } & \multicolumn{4}{|c}{ Our method } \\
& & & \multicolumn{2}{|c}{$\mu=0.9$} & \multicolumn{2}{c}{$\mu=1.1$} \\
\hline$N$ & CPU & Error & CPU & Error & CPU & Error & CPU & Error \\
6 & 0.4902 & $O\left(10^{-4}\right)$ & 0.0542 & $O\left(10^{-4}\right)$ & 0.0539 & $O\left(10^{-13}\right)$ & 0.0538 & $O\left(10^{-6}\right)$ \\
7 & 0.5519 & $O\left(10^{-5}\right)$ & 0.0597 & $O\left(10^{-6}\right)$ & 0.0589 & $O\left(10^{-13}\right)$ & 0.0598 & $O\left(10^{-6}\right)$ \\
8 & 0.7408 & $O\left(10^{-6}\right)$ & 0.0672 & $O\left(10^{-7}\right)$ & 0.0680 & $O\left(10^{-13}\right)$ & 0.0676 & $O\left(10^{-7}\right)$
\end{tabular}



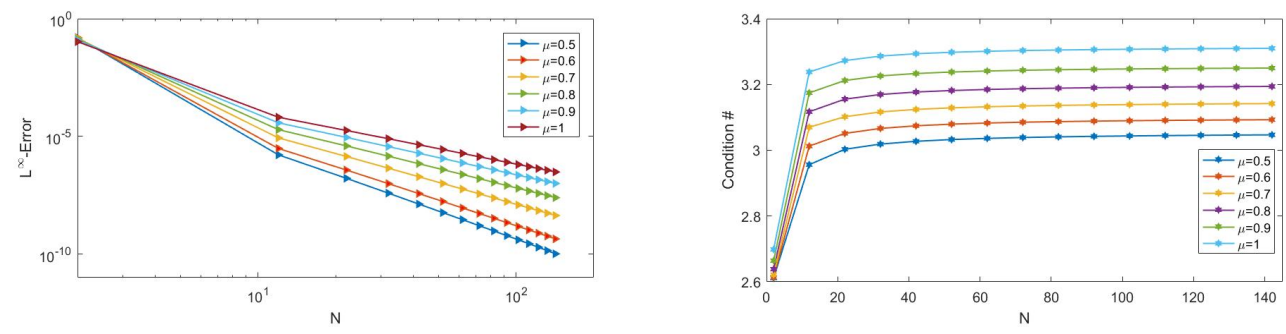

FIG. 7.4. The $L^{\infty}$-error of the numerical solution (left), and the corresponding condition number of the linear system (right) against $N$ for several values of $\mu$.
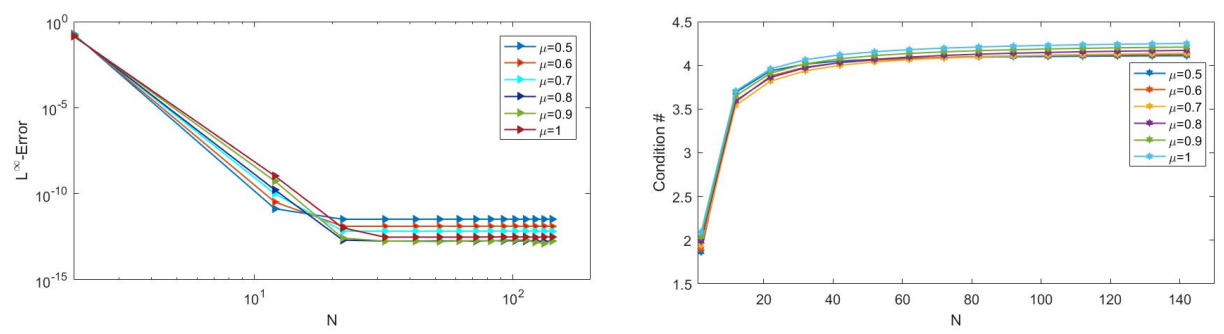

FIG. 7.5. The $L^{\infty}$-error of the numerical solution (left), and the corresponding condition number of the linear system (right) against $N$ for several values of $\mu$.

and in Figure 7.3, we present the numerical rates $r_{\mu, \alpha}$ and our expected rates $r_{\mu, \alpha}^{*}$ versus $N$ for these cases. It is observed that the desired convergence rates are obtained. We take the case $(1.2,1.5,6)$ which is solved in [8] using a fourth-order accurate scheme. Here, using our numerical approach, we obtain a convergence rate of $r_{\mu, 1.2}=\frac{2.6}{\mu}$. Finally, we choose a setting with $u(t)=t^{6}$ as the exact solution. In Table 7.5, we compare the error in the $L^{\infty}$-norm and the CPU time corresponding to the fractional spectral collocation method (FSCM) in [40] and our method.

In this example, we have tried to examine different cases of solutions including ones with various singularities at zero. We observe that the numerical results are well consistent with the theoretical analysis of the convergence rates. It is worth mentioning that these convergence rates are obtained for any linear/nonlinear FDEs.

EXAMPLE 7.2. In this example, to demonstrate the performance of the proposed methods, we investigate three multi-term FDEs. At first we consider [18]

$$
{ }_{0}^{c} D_{t}^{1.9} u(t)+(2+\sin (4 \pi t))_{0}^{c} D_{t}^{0.7} u(t)+(2+\cos t) u(t)=f(t), \quad t \in[0,1],
$$

with $u(0)=u^{\prime}(0)=1$ and the exact solution $u(t)=e^{t}+t^{6+\frac{4}{7}}+t^{5+\frac{4}{7}}$. Secondly, we consider [40]:

$$
\sum_{i=1}^{6}{ }_{0}^{c} D_{t}^{\alpha_{i}} u(t)=f(t), \quad\left(\alpha_{1}, \alpha_{2}, \alpha_{3}, \alpha_{4}, \alpha_{5}, \alpha_{6}\right)=\left(\frac{1}{5}, \frac{1}{3}, \frac{5}{7}, \frac{6}{5}, \frac{4}{3}, \frac{12}{7}\right),
$$

with $u(0)=u^{\prime}(0)=0$ and the exact solution $u(t)=t^{6+\frac{9}{17}}+t^{5+\frac{9}{17}}$.

In Figures 7.4-7.5, we display the errors in the $L^{\infty}$-norm of the numerical solutions (left) and the corresponding condition numbers of the system (right) versus $N$ and for several values of $\mu$ for the multi-term FDEs (7.1) and (7.2), respectively. We observe that the new scheme is 

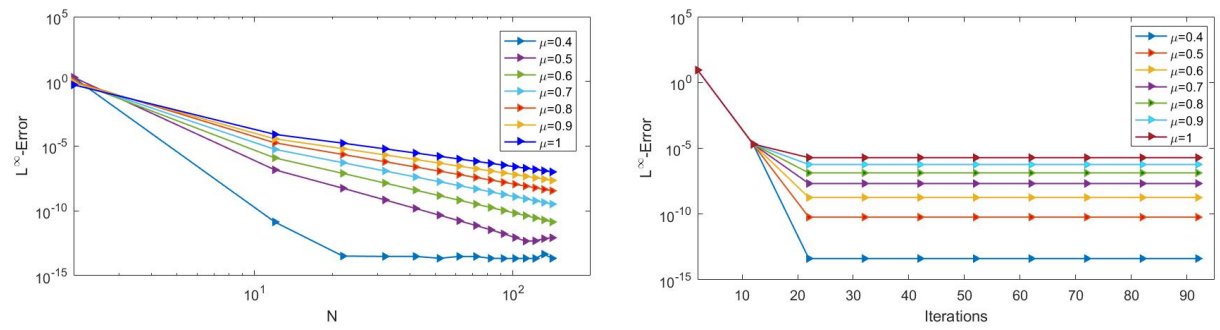

FIG. 7.6. The $L^{\infty}$-error of the numerical solution against $N$ (left), and $m$ (right) for several values of $\mu$.
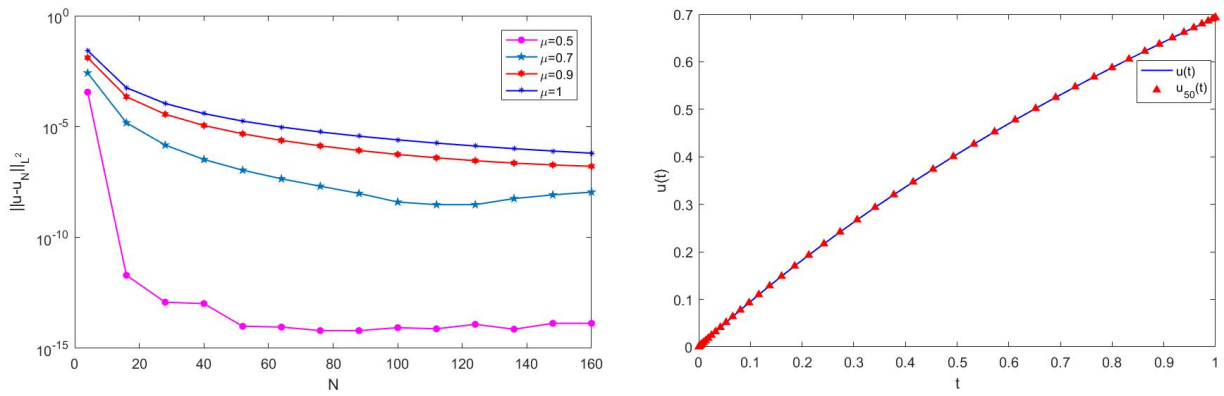

FIG. 7.7. The graph of the approximation errors $\left\|u-u_{N}\right\|_{L^{2}}$ versus $N$ for various $\mu$ (left), and the exact solution $u(t)$ and numerical solution $u_{50}(t)$ with $\mu=0.5$.

well-conditioned and the corresponding condition number of the linear system behaves like a constant (see $[18,40]$ for comparison). As a further observation, we notice that the new fractional pseudospectral schemes enjoy spectral accuracy.

It is noteworthy that the underlying solution of (7.1) and (7.2) are sufficiently smooth. Therefore, for a better understanding of the singularity, we examine the following non-linear FDEs

$$
{ }_{0}^{c} D_{t}^{1.5} u(t)+\left(1+e^{-1000 t^{2}}\right){ }_{0}^{c} D_{t}^{0.6} u(t)+\left(1+e^{-1000(t+0.2)^{2}}\right) u^{4}(t)=f(t),
$$

with initial conditions $u(0)=0, u^{\prime}(0)=1$ and the exact solution $u(t)=\sin (t)+t^{1.9}+t^{2.3}$, $t \in[0,1]$. In Figure 7.6, the convergence history of the $L^{\infty}$-errors versus $N$ with 25 iterations (left) and versus $m$ with $N=50$ (right) for different values of $\mu$ are presented. We can see that the numerical results are well consistent with the theoretical analysis and that our preconditioned system only requires few iterations.

EXAMPLE 7.3. Consider the following nonlinear FIDEs [34, 35]

$$
{ }_{0}^{c} D_{t}^{0.5} u(t)=\lambda(t) u(t)+f(t)+\sqrt{t}_{0} I_{t}^{1} u^{2}(t), \quad u(0)=0, \quad t \in[0,1],
$$

with

$$
\lambda(t)=(2-\ln (1+t))\left(\sqrt{t}+t^{1.5}\right), \quad f(t)=\frac{2 \operatorname{arcsinh}(\sqrt{t})}{\sqrt{\pi(1+t)}}-2 t^{1.5} .
$$

The exact solution of this problem is $u(t)=\ln (1+t)$. In order to distinguish the singularity index of $f(t)$, we apply the following algorithm: 


\section{ETNA}

Kent State University and

Johann Radon Institute (RICAM)

FRACTIONAL PSEUDOSPECTRAL METHODS FOR SOLVING FDES

TABLE 7.6

Our expected rates and the numerical rates with $N=70$ and various values of $\mu$.

\begin{tabular}{lllll} 
& $\mu=0.5$ & $\mu=0.7$ & $\mu=0.9$ & $\mu=1$ \\
\hline$r_{\mu, 0.5}$ & e. c & 4.3289 & 3.3815 & 3.0177 \\
$r_{\mu, 0.5}^{*}$ & e. c & 4.2857 & 3.333 & 3 \\
$r_{\mu, 0.5}$ & e. c & 2.8939 & 2.2385 & 2.0149 \\
$\hat{r}_{\mu, 0.5}^{*}$ & e. c & 2.8571 & 2.2222 & 2
\end{tabular}

TABLE 7.7

Approximation errors $\left\|u-u_{N}\right\|_{L^{2}}$ for our method with $\mu=\alpha=0.5$ and the method in [35].

\begin{tabular}{lllllll} 
& $N=10$ & $N=40$ & $N=70$ & $N=100$ & $N=130$ & $N=150$ \\
\hline Our method & $1.55 \mathrm{e}-08$ & $4.33 \mathrm{e}-13$ & $3.47 \mathrm{e}-13$ & $3.18 \mathrm{e}-13$ & $2.72 \mathrm{e}-13$ & $1.86 \mathrm{e}-13$ \\
Method in [35] & $3.62 \mathrm{e}-03$ & $5.64 \mathrm{e}-05$ & $1.05 \mathrm{e}-05$ & $3.615 \mathrm{e}-06$ & $1.64 \mathrm{e}-06$ & $1.07 \mathrm{e}-6$
\end{tabular}

Set the values of $[a, b]=[0,1]$ and $n \in \mathbb{N}$.

1. Evaluate the points $q_{j}=a+\frac{b-a}{n} j$, for $j=0,1, \ldots, n$.

2. Compute $p_{j}=\lim _{t \rightarrow 0} \frac{f(t)}{t^{q_{j}}}$, for $j=1, \ldots, n$.

3. Set $a=q_{k-1}$ and $b=q_{k}$, when $p_{k} \neq 0$ and $p_{i}=0$ for $i<k$.

4. Repeat Steps 1-3 until achieving the convergence criterion $\epsilon=|b-a|$.

In this example, we set $\epsilon=10^{-4}, a=0, b=1$, and $n=20$. With the above algorithm, we obtain $a=0.49984$ and $b=0.50016$. Thus, by adding the order of the FDEs, i.e., $\alpha=0.5$, the singularity index of the solution can be obtained. Using the generalized integral fractional pseudospectral scheme with collocation at the JG points and the introduced iterative method, the FIDE was solved in 10 iterations. It is seen from Figure 7.7 (left) that the errors in the $L^{2}$-norm decay algebraically for several different $\mu$ since the forcing term is not sufficiently smooth. The exact solution $u(t)$ and the numerical solution $u_{50}(t)$ are displayed in Figure 7.7 (right). In Table 7.6, we list our expected rates $r_{\mu, 0.5}$ and the numerical rates $r_{\mu, 0.5}^{*}$ for $N=70$ and some $\mu$. Again, we can observe that the desired rates of convergence are obtained. The approximation errors in the $L^{2}$-norm for our method and the method in [35] are compared in Table 7.7. The numerical results for this problem show that our schemes are more accurate than the fractional pseudospectral schemes introduced in [35] and are the same as the results in [34].

EXAMPLE 7.4. [12] Consider the following fractional oscillation equation

$$
{ }_{0}^{c} D_{t}^{\alpha} u(t)+u(t)=0, \quad t \in\left(0, t_{f}\right), \quad 1<\alpha<2,
$$

with initial condition $u(0)=1$ and $u^{\prime}(0)=0$. The analytical solution is $u(t)=E_{\alpha, 1}\left(-t^{\alpha}\right)$. We use the well-conditioned generalized differential fractional pseudospectral method at the FJGR points to solve this FDE. The approximation solutions for $\alpha=1.3,1.5,1.8,1.95$ and $t_{f}=20,50,100$ are given in Figure 7.8 with $N=300$. It is noteworthy that, contrary to the methods based on GIFs presented in [6,43], we have exponential convergence. To gain more insight, the problem was solved using the generalized integral fractional pseudospectral scheme with collocation at the FJGR points, and the errors $\left\|u-u_{N}\right\|_{L^{2}}$ versus $N$ for $\alpha=1.3,1.5,1.8,1.95, t_{f}=1$, and $\mu=0.5,0.7$, are presented in Figure 7.9.

EXAMPLE 7.5. As the last example, we consider the nonlinear FDEs [20]

$$
{ }_{0}^{c} D_{t}^{\alpha} u(t)=u_{1}\left(u_{0}-u(t)\right)^{4}, \quad t \in(0,50), \quad 0<\alpha<1,
$$



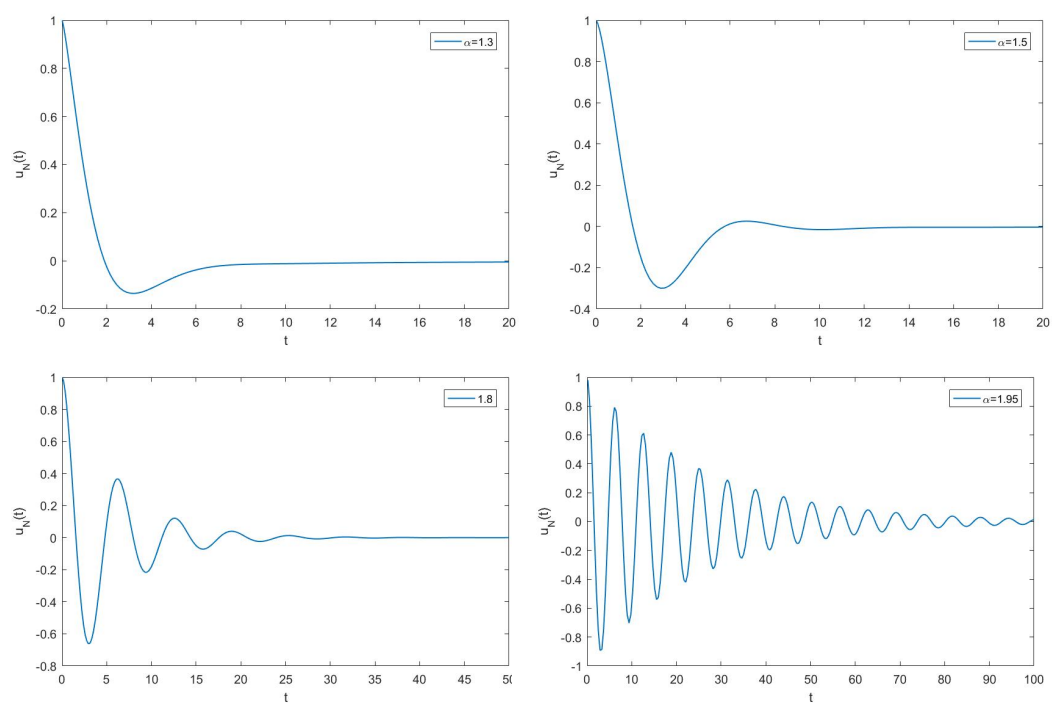

FIG. 7.8. Numerical solution $u_{300}(t)$ with $\alpha=1.3,1.5,1.85,1.95$ and $t_{f}=20,50,100$.
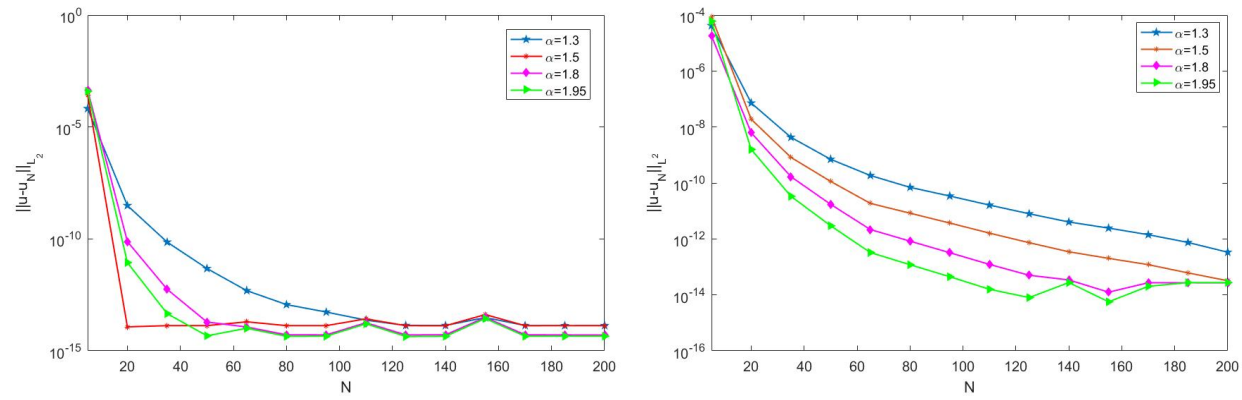
$($ right).

FIG. 7.9. The errors $\left\|u-u_{N}\right\|_{2}$ as a function of $N$ for $\alpha=1.3,1.5,1.8,1.95$ and $\mu=0.5$ (left), $\mu=0.7$

with initial condition $u(0)=0$. The solution has an asymptotic representation for $u(t)$ of the form

$$
\begin{aligned}
& u(t) \approx 2 u_{1} u_{0}^{4} \sqrt{\frac{t}{\pi}}, \quad t \ll 1 \\
& u(t) \approx u_{0}\left(1-\frac{1}{u_{1} \sqrt{\pi}}\right) t^{\frac{1}{8}} .
\end{aligned}
$$

This model for $\alpha=0.5$ arises in the process of cooling of a semi-infinite body by radiation. We apply the proposed method to solve this problem. In Figure 7.10, we display the asymptotic solution and the numerical solution obtained by the generalized differential fractional pseudospectral scheme for $u_{0}=u_{1}=1$ and for different values of $N$. The results can be compared with those in [20].

8. Conclusion. This paper provides the generalized fractional pseudospectral integration and differentiation matrices (GFPIMs and GFPDMs) in terms of fractional power Lagrange interpolation functions for solving fractional models such as fractional differential equations 


\section{ETNA}

Kent State University and

Johann Radon Institute (RICAM)

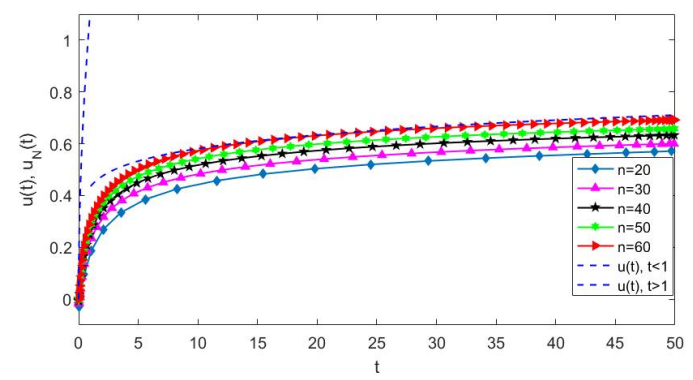

FIG. 7.10. Asymptotic solution and numerical solutions for different values of $N$.

(FDEs). One of the most important contributions of this work is that the new fractional pseudospectral scheme can effectively capture at least one end-point singularity factor of the solutions of fractional problems. We provide an efficient and stable approach using three-term formulas and Jacobi-Gauss-type quadrature to compute the GFPIMs and GFPDMs at the specific set of JG and FJGR points. Also, we use the remarkable property of the integral fractional pseudospectral scheme to preconditioning the systems resulting from the differential fractional pseudospectral scheme, and thus, we obtain well-conditioned systems. A rigorous convergence analysis and new convergence rates with detailed error estimates for the proposed fractional pseudospectral schemes are derived. The performance of the proposed methods is demonstrated by some numerical examples of FDEs. Furthermore, the numerical results verified the proposed spectral rate of convergence.

Acknowledgment. The authors declare no potential conflict of interest with respect to the research, authorship, and/or publication of this article.

\section{REFERENCES}

[1] R. A. Adams, Sobolev Spaces, Academic Press, New York, 1975.

[2] J. P. BerRut AND L. N. TREFETHEN, Barycentric Lagrange interpolation, SIAM Rev., 46 (2004), pp. 501517.

[3] G. W. Bohannan, Analog fractional order controller in temperature and motor control applications, J. Vib. Control., 14 (2008), pp. 1487-1585.

[4] P. Borwein, T. ERdÉLYI, AND J. Zhang, Müntz systems and orthogonal Müntz-Legendre polynomials, Trans. Amer. Math. Soc., 342 (1994), pp. 523-542.

[5] A. CARPINTERI AND F. MAINARDI, eds., Fractals and Fractional Calculus in Continuum Mechanics, Springer, Wien, 1997.

[6] S. Chen, J. Shen, AND L. L. WANG, Generalized Jacobi functions and their applications to fractional differential equations, Math. Comp., 85 (2016), pp. 1603-1638.

[7] Y. CHEN AND T. TANG, Convergence analysis of the Jacobi spectral-collocation methods for Volterra integral equations with a weakly singular kernel, Math. Comp., 79 (2010), pp. 147-167.

[8] M. CHEN AND W. DENG, Fourth order accurate scheme for the space fractional differential equations, SIAM J. Numer. Anal., 52 (2014), pp. 1418-1438.

[9] L. Debnath, Recent applications of fractional calculus to science and engineering, Int. J. Math. Math. Sci., 54 (2003), pp. 3413-3442.

[10] K. Diethelm, The Analysis of Fractional Differential Equations, Springer, Berlin, 2010.

[11] H. Ding, C. LI, AND Y. CHEN, High-order algorithms for Riesz derivative and their applications (II), J. Comput. Phys., 293 (2015), pp. 218-237.

[12] B. DUAN, Z. ZHENG, AND W. CAO, Spectral approximation methods and error estimates for Caputo fractional derivative with applications to initial-value problems, J. Comput. Phys., 319 (2016), pp. 108-128.

[13] S. ERFANI, E. BABOLIAN, AND S. JAVADI, Error estimates of generalized spectral iterative methods with accurate convergence rates for solving systems of fractional two-point boundary value problems, Appl. Math. Comput., 364 (2020), Art. Id. 124638, 15 pages. 
[14] S. ERfani, E. Babolian, S. Javadi, And M. Shamsi, Stable evaluations of fractional derivative of the Müntz-Legendre polynomials and application to fractional differential equations, J. Comput. Appl. Math., 348 (2019), pp. 70-88.

[15] S. ESMAeILI, M. ShAMSI, AND Y. LUCHKo, Numerical solution of fractional differential equations with a collocation method based on Müntz polynomials, Comput. Math. Appl., 62 (2011), pp. 918-929.

[16] B. GUO AND N. HEUER, The optimal rate of convergence of the p-version of the boundary element method in two dimensions, J. Numer. Math., 98 (2004), pp. 499-538.

[17] C. HUANG, Y. JiAO, L. L. WANG, AND Z. ZHANG, Optimal fractional integration preconditioning and error analysis of fractional collocation method using nodal generalized Jacobi functions, SIAM J. Numer. Anal., 54 (2016), pp. 3357-3387.

[18] Y. JIAO, L. L. WANG, AND C. HUANG, Well-conditioned fractional collocation methods using fractional Birkhoff interpolation basis, J. Comput. Phys., 305 (2016), pp. 1-28.

[19] A. A. Kilbas, H. M SRivastava, And J. J. Trujillo, Theory and Applications of Fractional Differential Equations, Elsevier, Amsterdam, 2006.

[20] H. Khosravian-Arab, M. Dehghan, And M. R. Eslahchi, Fractional Sturm-Liouville boundary value problems in unbounded domains: theory and applications, J. Comput. Phys., 299 (2015), pp. 526-560.

[21] M. KUMAR AND V. DAFTARDAR-GEJJI, A new family of predictor-corrector methods for solving fractional differential equations, Appl. Math. Comput., 363 (2019), Art. Id. 124633, 13 pages.

[22] X. LI AND C. XU, A space-time spectral method for the time fractional diffusion equation, SIAM J. Numer. Anal., 47 (2009), pp. 2108-2131.

[23] F. LiU, V. ANH, AND I. TURNER, Numerical solution of the space fractional Fokker-Planck equation, J. Comput. Appl. Math., 166 (2004), pp. 209-219.

[24] Z. MAO, S. CHEN, AND J. SHEN, Efficient and accurate spectral method using generalized Jacobi functions for solving Riesz fractional differential equations, Appl. Numer. Math., 106 (2016), pp. 165-181.

[25] G. V. Milovanovíc, Müntz orthogonal polynomials and their numerical evaluation, in Applications and Computation of Orthogonal Polynomials, W. Gautschi, G. H. Golub, and G. Opfer, eds., Internat. Ser. Numer. Math., 131, Birkhäuser, Basel, 1999, pp. 179-194.

[26] K. B. Oldham AND J. Spanier, The Fractional Calculus: Theory and Applications of Differentiation and Integration to Arbitrary Order, Dover, New York, 2006.

[27] J. Sabatier, O. P. Agrawal, And J. A. Tenreiro Machado, eds., Advances in Fractional Calculus: Theoretical Developments and Applications in Physics and Engineering, Springer, New York, 2007.

[28] J. Shen, T. TANG, AND L. L. WAng, Spectral Methods: Algorithms, Analysis and Applications, Springer, Berlin, 2011.

[29] J. SHEN AND Y. WANG, Müntz-Galerkin methods and applications to mixed Dirichlet-Neumann boundary value problems, SIAM J. Sci. Comput., 38 (2016), pp. A2357-A2381.

[30] F. SONG AND C. XU, Spectral direction splitting methods for two-dimensional space fractional diffusion equations, J. Comput. Phys., 299 (2015), pp. 196-214.

[31] X. TANG, Z. LIU, AND Y. HU, New results on pseudospectral methods for optimal control, Automatica J. IFAC, 65 (2016), pp. 160-163.

[32] X. TANG, Z. LIU, AND X. WANG, Integral fractional pseudospectral methods for solving fractional optimal control problems, Automatica J. IFAC, 62 (2015), pp. 304-311.

[33] X. TANG, Y. SHI, AND L. WANG, A new framework for solving fractional optimal control problems using fractional pseudospectral methods, Automatica J. IFAC, 78 (2017), pp. 333-340.

[34] X. TANG, Y. SHI, AND H. XU, Fractional pseudospectral schemes with equivalence for fractional differential equations, SIAM J. Sci. Comput., 39 (2017), pp. A966-A982.

[35] X. TANG AND H. XU, Fractional pseudospectral integration matrices for solving fractional differential, integral, and integro-differential equations, Commun. Nonlinear Sci. Numer. Simul., 30 (2016), pp. 248 267.

[36] A. WACHTER AND L. T. BIEGLER, On the implementation of an interior-point filter line-search algorithm for large-scale nonlinear programming, Math. Program., 106 (2006), pp. 25-57.

[37] B. J. West, M. Bologna, And P. Grigolini, Physics of Fractal Operators, Springer, NewYork, 2003.

[38] B. YUTTANAN AND M. RAZZAGHI, Legendre wavelets approach for numerical solutions of distributed order fractional differential equations, Appl. Math. Model., 70 (2019), pp. 350-364.

[39] M. ZaYernouri AND G. E. KARNiAda KIS, Fractional Sturm-Liouville eigen-problems: theory and numerical approximation, J. Comput. Phys., 252 (2013), pp. 495-517.

[40] - Fractional spectral collocation method, SIAM J. Sci. Comput., 36 (2014), pp. A40-A62.

[41] F. ZENG, Z. MAO, AND G. E. KARNIADAKIS, A generalized spectral collocation method with tunable accuracy for fractional differential equations with end-point singularites, SIAM J. Sci. Comput., 39 (2017), pp. A360-A383.

[42] F. ZENG, Z. ZHANG, AND G. E. KARNIADAKIS, A generalized spectral collocation method with tunable accuracy for variable-order fractional differential equations, SIAM J. Sci. Comput., 37 (2015), pp. A2710A2732. 
ETNA

Kent State University and

Johann Radon Institute (RICAM)

FRACTIONAL PSEUDOSPECTRAL METHODS FOR SOLVING FDES

[43] Z. ZHANG, F. ZENG, AND G. E. KARNIADAKIS, Optimal error estimates of spectral Petrov- Galerkin and collocation methods for initial value problems of fractional diferential equations, SIAM J. Numer. Anal., 53 (2015), pp. 2074-2096.

[44] T. ZHAO, Z. MAO, AND G. E. KARNIADAKIS, Multi-domain spectral collocation method for variableorder nonlinear fractional differential equations, Comput. Methods Appl. Mech. Engrg., 348 (2019), pp. 377-395. 